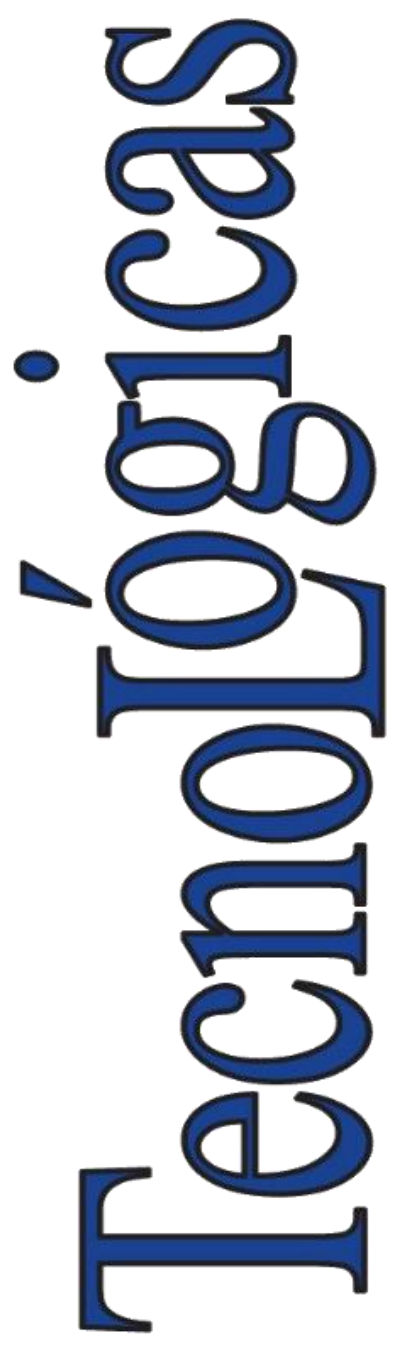

ISSN-p: 0123-7799 ISSN-e: $2256-5337$

Vol. 24, nro. 52, e2065, 2021

Recibido: 28 junio 2021 Aceptado: 18 noviembre 2021 Disponible: 16 diciembre 2021

CInstituto Tecnológico Metropolitano Este trabajo está licenciado bajo una Licencia Internacional Creative Commons Atribución (CC BY-NC-SA)

\section{Modelo de desarrollo para la construcción de aplicaciones móviles educativas}

\author{
Development Model for the Construction of Educational \\ Mobile Applications
}

\author{
(D) Jorge Luis Escobar-Reynel1; \\ (iD) Rubén Baena-Navarro²; \\ (iD Beatriz Giraldo-Tobón' ${ }^{3}$; \\ (iD) Mario Macea-Anaya ${ }^{4}$; \\ (D) Samir Castaño-Rivera ${ }^{5}$ \\ ${ }^{1}$ Universidad Internacional Iberoamericana, Universidad de Santander \\ Bucaramanga-Colombia, \\ jorge.escobar@cvudes.edu.co \\ 2 Universidad Cooperativa de Colombia, Montería-Colombia, \\ ruben.baena@campusucc.edu.co \\ ${ }^{3}$ Universidad de Santander, Bucaramanga-Colombia, \\ beatriz.tobon@cvudes.edu.co \\ ${ }_{4}$ Universidad de Córdoba, Montería-Colombia, \\ mariomacea@correo.unicordoba.edu.co \\ ${ }^{5}$ Universidad de Córdoba, Montería-Colombia, \\ sacastano@correo.unicordoba.edu.co
}

Cómo citar / How to cite

J. L. Escobar-Reynel; R. Baena-Navarro; B. Giraldo-Tobón; M. MaceaAnaya; S. Castaño-Rivera, "Modelo de desarrollo para la construcción de aplicaciones móviles educativas", TecnoLógicas, vol. 24, nro. 52, e2065, 2021. https://doi.org/10.22430/22565337.2065 


\title{
Resumen
}

Los dispositivos móviles promueven la construcción de saberes, fomentando el aprendizaje social y el intercambio de conocimientos. El aprendizaje móvil genera oportunidad de crecimiento y aplicación de prácticas innovadoras en los entornos educativos. El Gobierno colombiano, a través de estrategias de inversión que apuestan a la calidad educativa, dota de dispositivos móviles a las escuelas del país. No obstante, las aplicaciones móviles contenidas en estos dispositivos carecen de temáticas acordes al contexto y nivel educativo, diseño UX/UI y no promueven un mejoramiento del proceso de enseñanza-aprendizaje. El propósito de este trabajo fue presentar un modelo para el desarrollo de aplicaciones móviles educativas para docentes no programadores, que integre estrategias didácticas y metodológicas orientadas al mejoramiento académico. El desarrollo de este trabajo se apoyó en el uso de la investigación descriptiva con enfoque mixto, para hacer una interpretación de los resultados obtenidos y analizar la mediación de las aplicaciones móviles creadas bajo este modelo para coadyuvar en el proceso de enseñanza-aprendizaje. La investigación se llevó a cabo con docentes de diez instituciones educativas públicas, quienes presentaban desconocimiento y poca apropiación del aprendizaje móvil. Como resultado se proporcionó un modelo de desarrollo de aplicaciones móviles educativas, una contribución a la literatura existente en el contexto del aprendizaje móvil y el desarrollo de prácticas docentes innovadoras. Se concluye que el modelo de desarrollo de esta investigación puede ser utilizado para que profesores no desarrolladores puedan implementar aplicaciones móviles educativas que sirva como herramienta didáctica mediadora en el proceso enseñanza-aprendizaje en la educación primaria y secundaria.

\section{Palabras clave}

Dispositivos móviles, aprendizaje móvil, aplicaciones móviles, material didáctico, tecnología educativa.

\begin{abstract}
Mobile devices promote the construction of knowledge, fostering social learning and knowledge exchange. Mobile learning creates an opportunity for growth and the application of innovative practices in educational settings. The Colombian government has implemented investment strategies to improve educational quality and provided schools throughout the country with mobile devices. However, the mobile applications contained in these devices lack contents in accordance with student's context and educational level and UX/UI design; moreover, they do not promote an improvement in the teaching-learning process. This study presents a model that non-programming teachers can use to develop educational mobile applications, which integrates didactic and methodological strategies aimed at academic improvement. Descriptive research with a mixed-methods approach was employed here to interpret the results obtained and analyze the mediation of the mobile applications created adopting this model to support the teaching-learning process. Teachers from ten public educational institutions, who exhibited little appropriation and ignorance of mobile learning, participated in this study. As a result, a model to develop educational mobile applications was created, which represents a contribution to the existing literature in the field of mobile learning and the development of innovative teaching practices. It was concluded that the development model proposed in this paper can be used by non-developer teachers to implement educational mobile applications that serve as a mediating didactic tool in the teaching-learning process in primary and secondary education.
\end{abstract}

\section{Keywords}

Mobile device, mobile learning, mobile applications, courseware, educational technology. 


\section{INTRODUCCIÓN}

Con la integración de la tecnología educativa, los métodos de aprendizaje han cambiado, volviéndose más dinámicos e interactivos y el aprendizaje más intuitivo [1]. La integración de la tecnología móvil en la educación es una iniciativa muy importante del Gobierno colombiano, en cabeza del Ministerio de Tecnologías de la Información y las Comunicaciones (MinTic) [2].

En este sentido, Computadores para Educares uno de los programas sociales más relevantes propuestos por el gobierno de Colombia, porque ayuda a reducir la brecha digital y de conocimiento adquiriendo y utilizando tecnologías de la información y la comunicación (TIC) para los centros de educación pública del país [3]-[5].

En 2012, este programa lanzó por primera vez el Concurso Regional de Tabletas para Educar, concebido, de manera estratégica, para la apropiación pedagógica de dispositivos móviles digitales en entornos educativos oficiales con fines didácticos. Esta política donó más de 35000 tabletas para su uso en las secretarías de Educación de las entidades territoriales certificadas y no certificadas del país [6]. Según datos reportados por las distintas secretarías de educación de Colombia, sobre los estudiantes matriculados por el Ministerio de Educación Nacional, a diciembre de 2018 existía un computador de uso educativo por cada siete estudiantes, y una tableta de uso educativo por cada cinco estudiantes [7], [8]. La brecha en el uso de herramientas tecnológicas entre los estudiantes de las instituciones educativas oficiales en Colombia se ha reducido en gran medida, por lo que en 2010 solo había una herramienta tecnológica por cada veinte estudiantes, pero para 2018 la cantidad de estudiantes se redujo a solo siete. En los últimos ocho años, la contribución realizada para incrementar el uso de software superó el $50 \%$ [7]; por lo tanto, se puede afirmar que existen hardware que pueden utilizar las TIC para desarrollar procesos horizontales en el aula a través de los docentes [9], [10].

Por otro lado, cuando se hace referencia a la conectividad a Internet en instituciones educativas (IE), cabe recordar que en 2017 solo un $62 \%$ de las instituciones urbanas tenían acceso limitado a ella, mientras que apenas un $38 \%$ de las instituciones rurales regularmente tenían acceso al servicio [11], [12]. Por tal motivo, de la mano de las herramientas tecnológicas, debe estar un servicio de conectividad suficiente para la demanda de las IE, porque si bien el tener herramientas tecnológicas es un asunto muy importante, contar con un servicio de internet hace que el proceso de apropiación de las TIC en la educación sea más eficiente; lamentablemente en el país los proyectos de conectividad no han ido paralelos a la adquisición de herramientas, por lo que ha tomado más tiempo para ser implementado en los porcentajes que se requieren [13].

En este sentido, el problema central de esta investigación radica en que las aplicaciones móviles educativas contenidas en hardware (tabletas), donadas por el Estado, no abordan temáticas acordes al contexto y nivel educativo de los estudiantes ni generan una influencia motivacional; además, el componente lúdico es limitado, no mejoran el nivel de atención en clase, visualmente sus contenidos son poco agradables y sus interfaces gráficas de usuario no permiten el acceso inmediato a la información, no poseen un diseño usable y responsivo, no fomentan el aprendizaje autodirigido y, en general, no promueven un mejoramiento del proceso enseñanza-aprendizaje [14]-[16].

De lo anterior la importancia de construir este modelo de desarrollo de aplicaciones móviles educativas para docentes no desarrolladores, que promuevan contenidos digitales que se encuentren contextualizados y puedan ponerse en práctica al interior de las aulas de clase, generando procesos de capacitación a los docentes para que tengan la posibilidad de diseñar y desarrollar sus propias aplicaciones, para así contribuir con el mejoramiento académico de sus estudiantes. 
Con el firme apoyo del Ministerio de Educación, se debe dar prioridad a los servicios de interconexión escolar para cubrir las instituciones educativas de manera oportuna y efectiva.

Los recursos de hardware a menudo se deterioran debido a la falta de uso, porque en la mayoría de las instituciones educativas la conexión está vacía o es intermitente [7], [17].

De igual manera es importante monitorear y asegurar que el contenido digital que se brinda a las instituciones educativas al donar computadoras y tabletas sea acorde con las necesidades ambientales y académicas de cada región para que los recursos técnicos se pueden utilizar de manera completa y adecuada [2], [18].

Existen diversas investigaciones sobre aprendizaje móvil para determinar cómo se utilizan estos dispositivos en todas las áreas temáticas [19]-[23], sin embargo son pocos los modelos de enseñanza que definen un marco de trabajo para la construcción de aplicaciones móviles a la medida de las concepciones de aprendizaje (blended learning, e-learning, m-learning, virtual learning environments, ICT-enhanced), pero sobre todo en docentes que no poseen las competencias requeridas para desarrollar software [24]-[28]. El aprendizaje móvil puede ser una herramienta eficaz para respaldar la integración tecnológica significativa y, en este sentido, se han desarrollado trabajos que buscan mejorar la enseñanza y el aprendizaje. En la Figura 1 se muestra el cálculo de la frecuencia de ocurrencias y coocurrencia de palabras clave de 30 trabajos de investigación entre los años 2016 y 2021, cargados en la herramienta VOSviewer. Para ubicar palabras clave en el mapa, se utilizó la tecnología de visualización de similitud (VOS) [29], para identificar y visualizar de los grupos temáticos tomados de Scopus, Science Direct y PubMed. La cadena de búsqueda utilizada con las palabras es: "mobile learning", "e-learning", "teaching" y "mobile technology".

Los colores de la Figura 1 próximos al rojo muestran las zonas de mayor densidad de coocurrencias de palabras clave; los colores próximos al amarillo y verde muestran las zonas de menor densidad de coocurrencias de palabras clave.

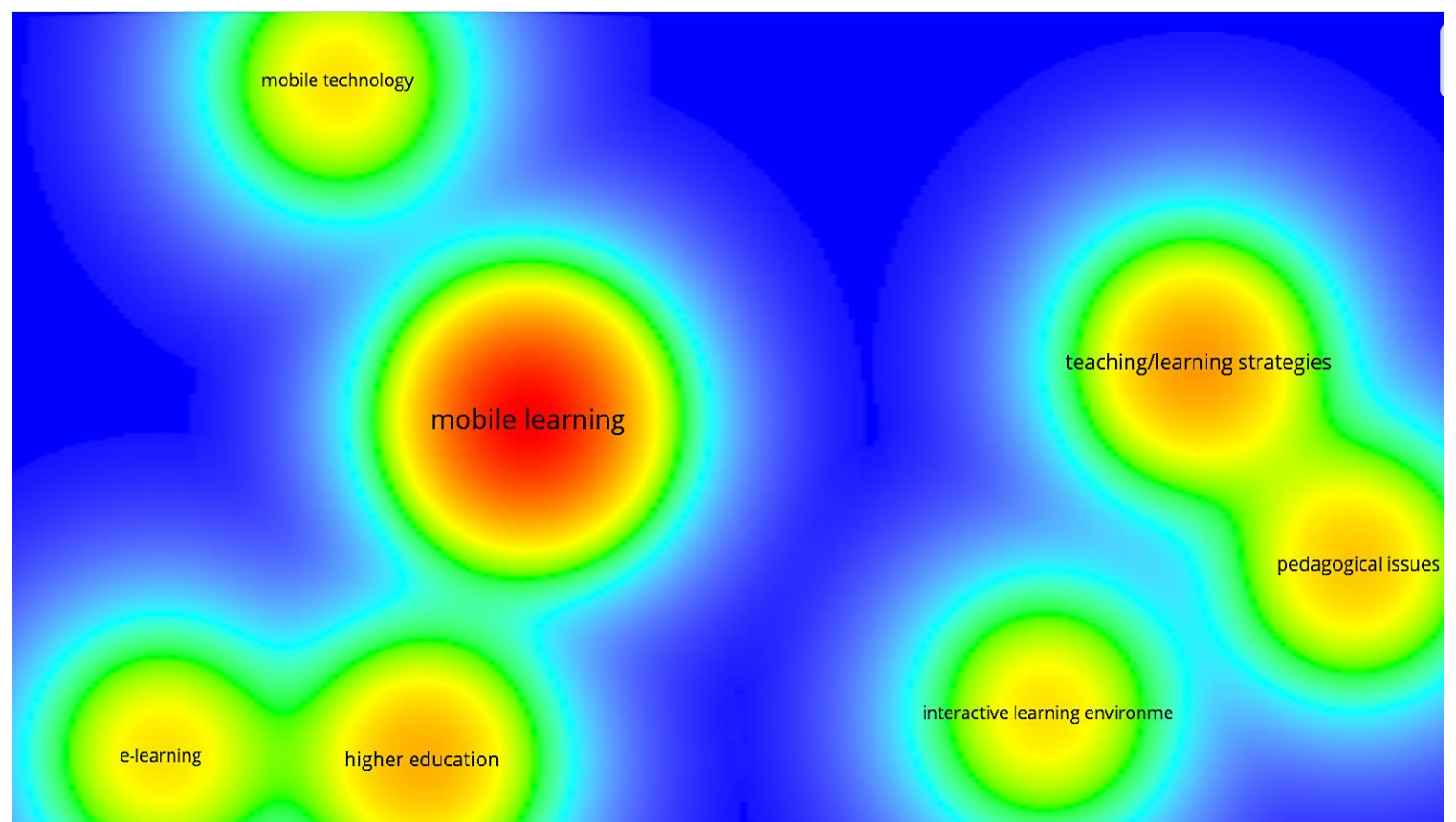

Figura. 1. Mapa bibliométrico de densidad en el que se representaron siete palabras clave analizadas Fuente: elaboración propia. 
Se puede apreciar una amplia investigación de aprendizaje móvil en educación superior y por ello, desde esta perspectiva, este trabajo presenta un modelo de desarrollo que permite la integración de aspectos docentes y técnicos en el desarrollo de aplicaciones de educación móvil para dar respuesta a las necesidades del entorno educativo y combinarlas como intermediario en el proceso de enseñanza-aprendizaje de las escuelas primarias y secundarias en las instituciones educativas públicas colombianas.

De igual forma, el estudio avanzó en la implementación de una mejor práctica docente que involucra aspectos tecnológicos, pedagógicos, cognitivos y sociales en el proceso de enseñanzaaprendizaje, impactando positivamente el fortalecimiento de competencias en las distintas áreas del saber a través de la transversalización con aplicaciones móviles educativas diseñadas y desarrolladas a la medida por docentes no programadores y contextualizadas a las necesidades del entorno. La investigación promueve que el docente con experticia en pedagogía se involucre en tareas de arquitectura de la información (sketch, wireframes y mockup) y desarrollo de software a través del uso de la herramienta App Inventor, permitiendo establecer así un modelo en donde la construcción de recursos educativos digitales no es labor especifica de ingenieros de software o programadores, sino de transversalización curricular para potenciar el robustecimiento del servicio educativo.

A día de hoy, el software es utilizado en diferentes contextos vitales para el ser humano, es por ello que se hace necesario que este pueda cumplir con el fin para el que fue creado y satisfacer la necesidad de los usuarios [30]. Abordando la temática de calidad de software, es imperativo hallar diferentes aspectos. Uno de los más relevantes, y que hace parte de esta temática es la usabilidad, la cual se expresa como un atributo, pero la atención prestada a la funcionalidad y su relación con aspectos de calidad es prácticamente nula, por lo que se olvida que a través de esta se puede alcanzar y desarrollar un sistema de mayor calidad [30], [31].

\section{MATERIALES Y MÉTODOS}

\subsection{Modelo propuesto}

Este trabajo se apoyó en el uso de la investigación descriptiva con un enfoque mixto [32] para interpretar los resultados obtenidos una vez fue puesto en práctica por parte del docente el modelo de desarrollo de aplicaciones móviles educativas de esta investigación (Figura 2), como intervención en un proceso de aprendizaje en la educación básica y media.

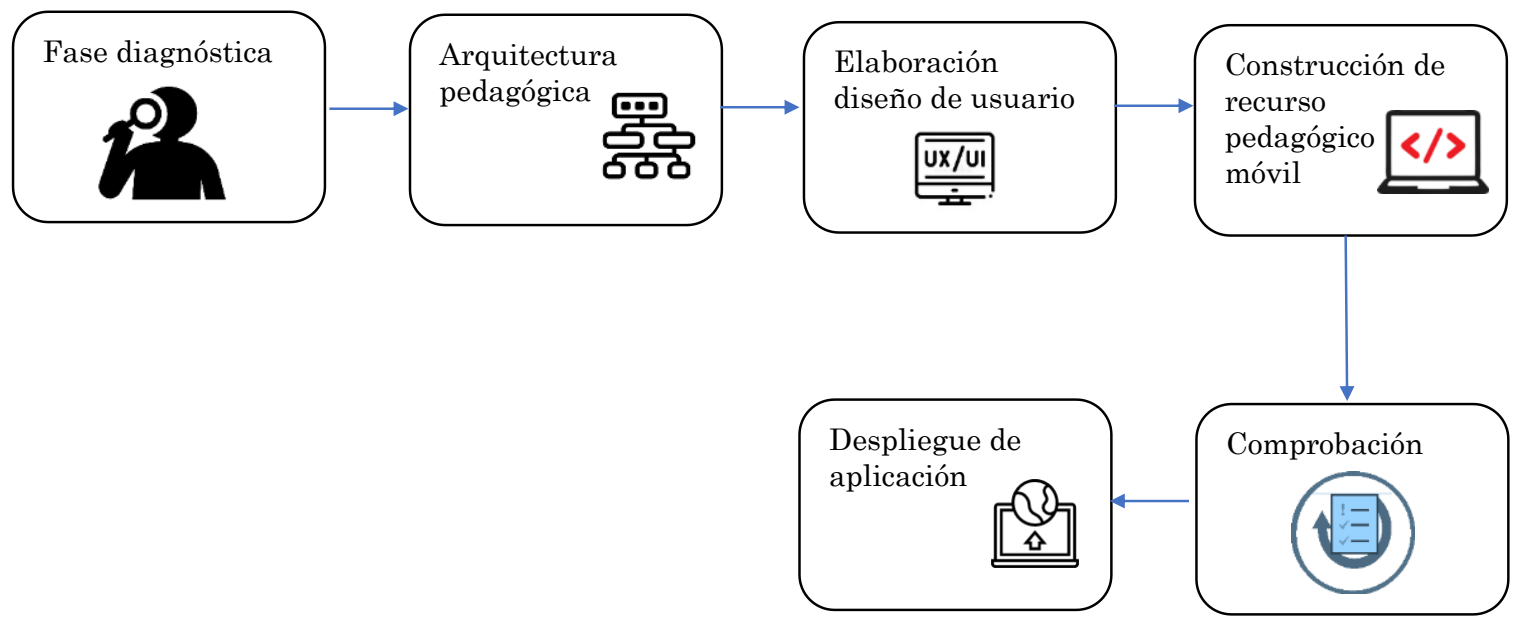

Figura 2. Modelo de desarrollo de aplicaciones móviles educativas. Fuente: elaboración propia. 
A partir del muestreo aleatorio estratificado con afijación proporcional que tiene una confiabilidad del $90 \%$ [33], se seleccionaron diez instituciones educativas públicas entre las diferentes regiones del país y cuarenta docentes de las distintas áreas del saber de nivel básica primaria y secundaria, con amplia experiencia en el sector educativo oficial.

Las variables trabajadas en esta investigación fueron: fortalecimiento del proceso enseñanza-aprendizaje en las instituciones educativas oficiales objetos de la investigación, confiabilidad del modelo de desarrollo propuesto en el diseño y construcción de aplicaciones móviles educativas usables, nivel de uso, apropiación y mejoramiento de estas por parte de los docentes en las distintas áreas de desempeño intervenidas a través de este modelo de desarrollo.

El modelo presentado representó un aporte que coadyuvó a que profesores no especializados en desarrollo de software pudieran construir aplicaciones educativas que apoyaran el proceso de enseñanza-aprendizaje que involucrara la aplicación de TIC móviles para cualquier tipo de plataforma (Android, iOS, entre otras), distinto a los modelos donde se emplean apps móviles que no son desarrolladas a la medida [34], [35]. Su objetivo es definir la metodología de desarrollo de software que tiene las características de componentes instruccionales (contenidos, técnicas de diseño, desarrollo, pruebas y despliegue) que permita la construcción de aplicaciones educativas para dispositivos móviles a docentes no desarrolladores de software, como estrategia para integrarlos eficazmente en el entorno educativo.

\subsection{Fases del modelo de desarrollo propuesto}

A continuación, se presenta una serie de fases que los equipos de trabajo de profesores pueden seguir para desarrollar aplicaciones móviles educativas.

\subsubsection{Fase diagnóstica}

En esta fase se concretan algunas características de usuarios finales, con su problemática de aprendizaje y los requerimientos técnicos acorde al contexto de la institución educativa que permitan el desarrollo de aplicaciones móviles. La Figura 3 muestra las etapas de esta fase.

En la etapa de concretar la problemática de aprendizaje [36], se recolecta información con técnicas de observación, entrevistas a estudiantes y docentes, y revisión documental, entre otros, esto con el objetivo de identificar, tanto la problemática de aprendizaje, como el área del conocimiento en la que se está presentando, lo que permite delimitar las competencias a desarrollar con ayuda de aplicaciones móviles.

Luego, en la etapa de caracterización del grupo de estudiantes, se identifican características como: edad cronológica, curso, estrato socioeconómico, disponibilidad, manejo de recursos tecnológicos y nivel de apropiación de herramientas TIC [37].

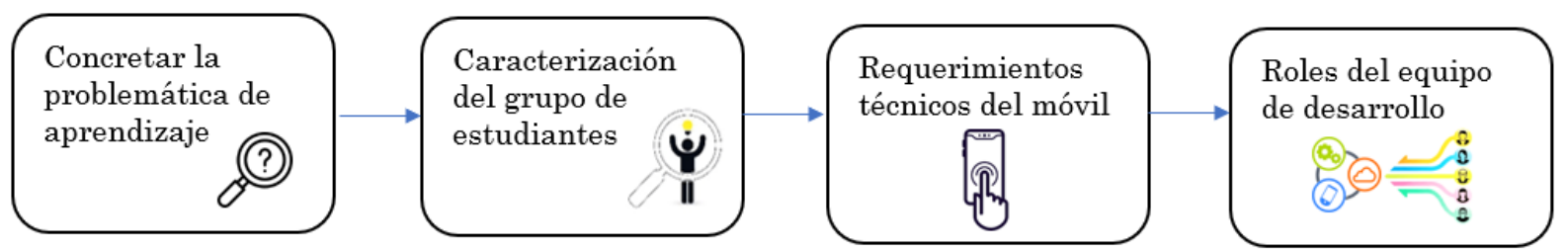

Figura 3. Etapas de la fase diagnóstica. Fuente: elaboración propia. 
Estos aspectos se tienen en cuenta para identificar las características poblacionales del contexto en el que se pretende implementar la aplicación móvil, de tal forma que se adapte a los mismos.

Después, viene la etapa para determinar los requerimientos técnicos del móvil [38], donde se realiza un diagnóstico tecnológico del hardware y el software de los dispositivos móviles con los que cuenta la institución educativa. Los aspectos técnicos para tener en cuenta son: capacidad de almacenamiento, memoria, procesamiento, puertos de conexión, sistema operativo, tamaño de la pantalla.

Posteriormente, en la etapa de roles del equipo de desarrollo, se designan las funciones de un equipo multidisciplinario compuesto por: líder (dirige todo el proceso, reconoce completamente todas las fases del modelo de desarrollo y posee suficientes competencias pedagógicas y tecnológicas ; también se encarga de ejecutar pruebas unitarias y de contexto, y debe difundir los resultados de estas a los demás integrantes del equipo de trabajo); docente de tecnología e informática de la institución educativa (se encarga de instruir a los estudiantes y docentes de otras áreas para que desarrollen habilidades básicas en programación, utilizando bloques lógicos y herramientas de pseudocódigo [39]); semillero de investigación (grupo de estudiantes de los grados 9 a 11, con conocimientos en el uso de herramientas de programación, quienes se encargan de hacer el acompañamiento respectivo al docente de área para que se construya la aplicación móvil); docente de área (identifica la necesidad y recibe el acompañamiento constante del equipo de trabajo) [40].

\subsubsection{Fase de arquitectura pedagógica}

Realizada la fase anterior, se define la arquitectura o diseño pedagógico del contenido y su objetivo de aprendizaje, la metodología, las actividades y las secuencias didácticas que serán implementadas en la aplicación educativa móvil. Las etapas para el desarrollo de esta fase se muestran en la Figura 4.

En la etapa de definir el perfil del estudiante, se establece el nivel de desarrollo cognitivo a través de la identificación de los procesos mentales y estilos de aprendizaje, conocimientos previos, discapacidades o limitaciones cognitivas, habilidades y competencias, de tal forma que se puedan definir perfiles de comportamiento en común para contextualizar los contenidos a los intereses y necesidades del grupo de estudiantes con quienes se implementa la propuesta de la aplicación móvil educativa.

Sigue la etapa de planeación de objetivos de aprendizaje, que se establecen con la respuesta a los siguientes interrogantes: ¿cuáles son los efectos formativos de los estudiantes al desarrollar las actividades propuestas en la aplicación móvil? o ¿qué competencias se desea fortalecer? Esta etapa permite entender hasta qué punto se pretende abarcar en la aplicación móvil y por supuesto identificar cuáles son las actividades y recursos necesarios para el desarrollo de esta.

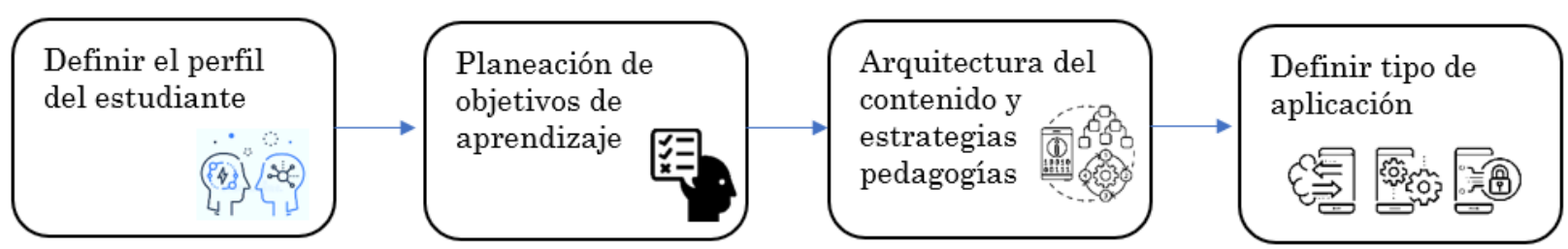

Figura 4. Etapas de la fase de arquitectura pedagógica. Fuente: elaboración propia. 
Más adelante viene la etapa de arquitectura del contenido y estrategias pedagógicas, que plantea la estrategia pedagógica con su metodología de trabajo, es decir, se definen los momentos del proceso enseñanza-aprendizaje enunciados en la metodología de trabajo del área y los momentos de desarrollo de esta. Aquí se deben seleccionar los contenidos y recursos necesarios para el logro del aprendizaje, tales como: texto, audio, sonido, imágenes, video, animaciones, entre otros.

Definidas las etapas anteriores de esta fase, continua la etapa de definir el tipo de aplicación móvil que se pretende desarrollar, atendiendo las diferentes características de las mismas y a las necesidades del contexto [41], [42], como se muestra en la Tabla 1:

Tabla 1. Tipos de aplicaciones móviles educativas. Fuente: elaboración propia.

Nativas

Híbridas o con conectividad
Aplicaciones desarrolladas para los sistemas operativos IOS o Android.

Potencian los recursos de los dispositivos en donde se utiliza la aplicación móvil: GPS, sensores, giroscopio, cámara, almacenamiento y demás.

Utilizar este tipo aplicación cuando se requiera de contenidos dinámicos o interactivos sin necesidad de ser instalada en el dispositivo móvil.

Utiliza algunos recursos nativos, pero también otros incrustados de sitios o plataformas webs externas.

Para ser usable necesita de una buena conectividad.

Utilizar este tipo aplicación cuando se requiera de contenidos dinámicos o interactivos que necesiten datos y si el contexto lo permite.

\subsubsection{Fase de elaboración de diseño usuario}

En esta fase se elaboran los diseños visuales acordes a la arquitectura de información de la aplicación móvil educativa, definida en las fases anteriores, se elaboran elementos tales como: sketch (boceto inicial), wireframes (mapa navegación y prototipo de interfaz de usuario) y mockup (diseño final de interfaz de usuario) [43]-[47] en los que se muestran de forma clara la organización de los recursos y elementos constituidos en la aplicación. Sus etapas se presentan en la Figura 5.

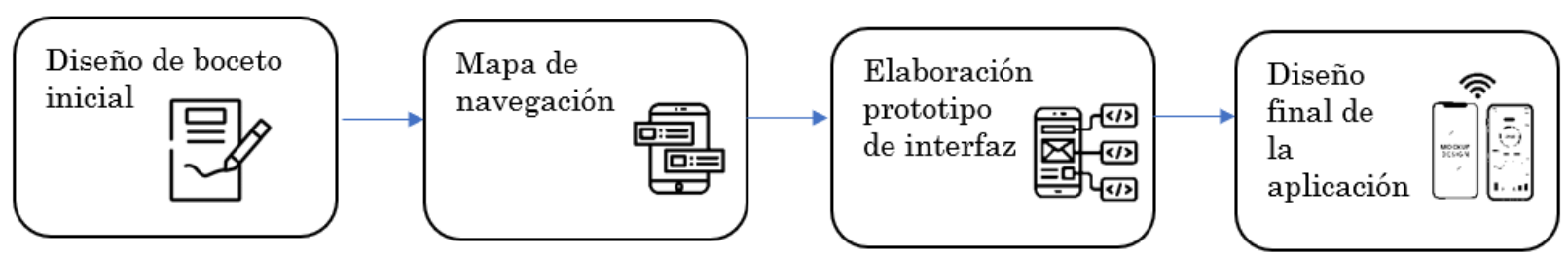

Figura 5. Etapas de la fase de elaboración de diseño de usuario. Fuente: elaboración propia.

\subsubsection{Construcción de recurso pedagógico móvil}

Luego de la fase de elaboración de diseño usuario, se desarrolla la aplicación educativa en la fase construcción de recurso pedagógico móvil, utilizando la frase What You See Is What You Get (Wysiwyg) [48]-[52], que facilita a los profesores no desarrolladores escribir lo que quieren ver, permitiendo crear aplicaciones móviles educativas a partir de los bocetos definidos en la fase anterior. La herramienta App Inventor permite su uso, esto corresponde al desarrollo front-end; sus funcionalidades back-end son codificadas/desarrolladas en esta herramienta de desarrollo, la cual utiliza un lenguaje de programación basado en bloques (blocks and logic) [39], [53], lo que coadyuva a los docentes que desconocen de programación de aplicaciones móviles [54]. A partir de la versión 0.9 del complemento MIT App Inventor se 
pueden crear aplicaciones móviles ajustadas al sistema operativo iOS [55]. Las etapas de esta fase se presentan en la Figura 6.

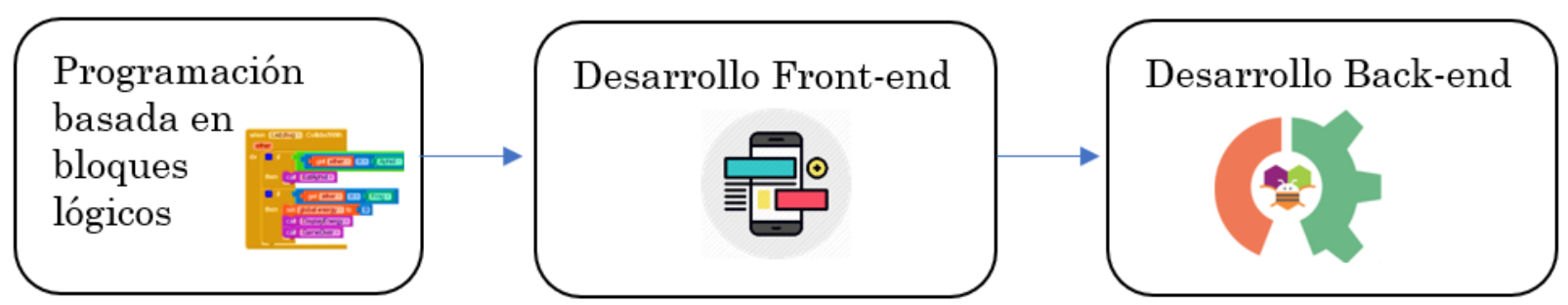

Figura 6. Etapas fase de desarrollo. Fuente: elaboración propia.

\subsubsection{Comprobación}

Desarrollada la aplicación móvil, se pone a prueba su usabilidad [56]-[59]. Se busca detectar errores de ejecución de la aplicación, de arquitectura de la información, factores que afecten la presentación de la aplicación y la satisfacción del usuario [60]. En este sentido, el proceso se apoya en la aplicación de instrumentos de información, tales como rúbricas, listas de chequeo para realizar la fase de comprobación.

\subsubsection{Despliegue de la aplicación}

Evaluada la funcionalidad y usabilidad de la aplicación móvil desarrollada con este modelo, se lleva a cabo el despliegue [61] para que pueda usarse en el desarrollo de las clases.

Cabe resaltar que la funcionalidad y la usabilidad no tienen como meta, en ningún momento, reemplazar el papel del docente, sino, por el contrario, que estas sean una herramienta de apoyo para fortalecer los procesos de enseñanza-aprendizaje orientados por él. Esta fase incluye la documentación, generación de manuales e inclusión de la aplicación educativa en la tienda de aplicaciones.

\subsection{Diagnóstico de competencias de aprendizaje móvil de los docentes}

Para la implementación del modelo de desarrollo presentado, se caracterizó el contexto en donde se aplicará para reconocer los puntos de partida que esta oferta y dónde se quiere llegar con el uso de la estrategia. Para ello se aplicaron encuestas, permitiendo una recogida de datos de manera concreta y directa; además se empleó un instrumento con el objetivo de identificar el nivel de apropiación de los cuarenta docentes que construyeron las aplicaciones móviles con este modelo, con relación al uso de aplicaciones móviles como herramientas didácticas mediadoras en el proceso enseñanza-aprendizaje.

La confiabilidad del cuestionario se realizó con método de coeficiente a [62], el cual está influenciado por las características propias del instrumento, por ejemplo, número de ítems y por la proporción de la varianza total del test debida a la covariación de los ítems [63], [64]; también está influido por aspectos estadísticos como el error muestral [64]. Como cualquier otra estimación estadística realizada en una muestra, su correspondiente valor poblacional puede variar en alguna magnitud. Para el cálculo del alfa de Cronbach se empleó en (1), [62]:

$$
\propto=\frac{K}{K-1}\left[1-\frac{\sum V i}{V t}\right]
$$


En donde:

$a=$ Alfa de Cronbach.

$\mathrm{K}=$ Número de ítems.

$\mathrm{Vi}=$ Varianza de cada ítem.

$\mathrm{Vt}=$ Varianza del total.

Los resultados para el cálculo del coeficiente alfa de Cronbach se detallan en la Tabla 2. Según este método, cuanto más se aproxime a su valor máximo, 1, mayor es la fiabilidad de la escala [63]. Para el caso de esta investigación, el resultado es de 0.952, lo cual significa que esta estadística se encuentra dentro del límite de la fiabilidad y consistencia de los ítems entre sí.

Tabla 2. Estadísticas del instrumento Fuente: elaboración propia.

\begin{tabular}{lccccccc}
\hline & Media & Mínimo & Máximo & Rango & $\begin{array}{c}\text { Máximo / } \\
\text { Mínimo }\end{array}$ & Varianza & $\begin{array}{c}\text { N de } \\
\text { elementos }\end{array}$ \\
\hline Medias de elemento & 3.182 & 2.175 & 4.525 & 2.350 & 2.080 & 0.401 & 25 \\
Varianzas de elemento & 1.356 & 0.358 & 1.999 & 1.641 & 5.580 & 0.163 & 25 \\
Covarianzas entre elementos & 0.586 & 0.072 & 1.549 & 1.621 & -21.571 & 0.088 & 25 \\
Correlaciones entre elementos & 0.443 & 0.045 & 0.858 & 0.902 & -19.189 & 0.032 & 25 \\
\hline
\end{tabular}

\section{RESULTADOS}

Con el diseño e implementación de un instrumento adaptado a los docentes se busca conocer la frecuencia con que ellos usan las aplicaciones móviles como herramientas didácticas mediadoras en el proceso enseñanza-aprendizaje; a partir de los resultados arrojados por este instrumento diagnóstico, se consolidan las bases para la puesta en marcha de la estrategia tecno-pedagógica basada en el modelo de desarrollo de aplicaciones móviles educativas. En particular, la fase diagnóstica inicial permitió establecer el nivel de uso y su apropiación en la educación básica y media, y así tener un referente para medir el impacto de la implementación del modelo al final del proceso investigativo. En este sentido, los resultados presentados en las Tablas 3 y 4. (La Tabla 3 hace referencia a tipos de aplicaciones y la Tabla 4 a funcionalidades que podrían tener cualquiera de esos tipos) evidencian que hay un desconocimiento generalizado por parte de los participantes en las posibilidades que ofrecen las aplicaciones móviles para dinamizar las actividades orientadas a favorecer las competencias digitales en los estudiantes. Aunado a lo anterior, se comprueba la necesidad de formar a los docentes en el uso de los recursos y herramientas digitales. 
Tabla 3. Instrumento diagnóstico a docentes con relación a acciones trabajadas con aplicaciones móviles Fuente: elaboración propia.

Criterio evaluado al docente con relación a las acciones trabajadas con aplicaciones móviles

Aplicaciones para buscar y filtrar

Aplicaciones para conocer y construir

Aplicaciones para aplicar y crear

Aplicaciones para analizar

Aplicaciones para colaborar, conectar y compartir
Resultado

El $48.8 \%$ de los encuestados expresó que ocasionalmente hacen uso de la búsqueda de información por medio de las aplicaciones móviles, lo cual demuestra que, con relación a las competencias digitales, este gran porcentaje de la muestra se encuentra en el nivel explorador.

Por su parte, el $24.4 \%$ de la muestra dijo que frecuentemente hacen la busque de información por medio de las aplicaciones móviles.

Tan solo el $7.3 \%$ de los docentes coinciden en que muy frecuentemente utilizan las aplicaciones para la búsqueda de información.

El $48.8 \%$ de los encuestados manifestó que frecuentemente han utilizado alguna aplicación para realizar algún tipo de procedimiento, mientras que $19.5 \%$ ha utilizado ocasionalmente, o muy frecuentemente, estas aplicaciones para realizar labores en sus instituciones educativas; finalmente, entre el $2.4 \%$ y el $9.8 \%$ nunca, o casi nunca, realizan trabajos apoyados en la utilización de aplicaciones móviles.

El 39 \% de los encuestados hacen algún tipo de usos de las aplicaciones móviles para desarrollar actividades, mientras que el $17.1 \%$ expresó que frecuentemente las utilizan para apoyar sus trabajos, y entre el $9.8 \%$ y el $29.3 \%$ manifestaron que nunca o casi nunca las utilizan para apoyar sus labores institucionales.

El $39 \%$ y el $26.8 \%$, ocasional o frecuentemente utilizan las aplicaciones móviles para el desarrollo de algún tipo de actividad descrita para este apartado, mientras que entre el $17.1 \%$ y el $7.3 \%$ nunca o casi nunca las utilizan, y solo el $9.8 \%$ lo hacen muy frecuentemente. Esto indica que los docentes no están aprovechando estas aplicaciones móviles para el apoyo de sus actividades, dejando de explorar y apropiar este recurso tecnológico disponible para el mejoramiento de sus procesos de enseñanza-aprendizaje con los estudiantes.

El $39 \%$ de los encuestados respondió que frecuentemente hacen usos de las aplicaciones móviles para desarrollar alguna de las actividades descritas.

Por su parte, el 39 \% de los encuestados manifestó que ocasionalmente utilizan estas herramientas para el desarrollo de sus labores, mientras que el $12.2 \%$ hace uso de ellas muy frecuentemente; y finalmente, entre el $7.3 \%$ y el $2.4 \%$, expresaron que nunca o casi nunca utilizan este tipo de aplicaciones móviles para hacer alguna de estas actividades. 
Tabla 4. Instrumento diagnóstico a docentes con relación a acciones trabajadas con funcionalidades de aplicaciones móviles. Fuente: elaboración propia.

\begin{tabular}{l|}
\hline $\begin{array}{l}\text { Criterio evaluado al } \\
\text { docente con relación a } \\
\text { las aplicaciones } \\
\text { móviles }\end{array}$ \\
\hline $\begin{array}{l}\text { Funcionalidad de redes } \\
\text { sociales }\end{array}$
\end{tabular}

Funcionalidad de reconocimiento de voz

Funcionalidad de cloud computing

Funcionalidad de realidad aumentada

Funcionalidad de mapas

Funcionalidad de sistema de posicionamiento global (GPS)

Funcionalidad para lectores de libros electrónicos (e-books)

Funcionalidad con wifi

Funcionalidad con Bluetooth

Funcionalidad con cámara

Funcionalidad de grabadora de sonidos

Funcionalidad de mensajería instantánea Funcionalidad de carácter enciclopédico, diccionario o de consulta

Funcionalidad de brújula

Funcionalidad de calculadora

Funcionalidad de agendas electrónicas

Funcionalidad de juegos

Funcionalidad de multimedia

Funcionalidad de pantalla táctil

Funcionalidad de manos libres
Resultado

El $48.8 \%$ de los docentes utilizan frecuentemente las redes sociales como apoyo a sus procesos pedagógicos; el $26.8 \%$ hace uso de ellas muy frecuentemente; el $14.6 \%$, ocasionalmente; y el $9.8 \%$, nunca o casi nunca.

El $36.6 \%$ de los encuestados respondió que ocasionalmente hacen uso de estas aplicaciones para abordar los procesos de enseñanza con los estudiantes que presentan algún tipo de condición o dificultad motriz; el $44 \%$ manifestó que nunca o casi nunca hacen uso de ellas; y el $17.5 \%$ aseguró que frecuente o muy frecuentemente realizan actividades con el apoyo de estas aplicaciones.

El $24.4 \%$ realiza esta acción ocasionalmente; el $26.8 \%$, lo hace frecuentemente; el $14.6 \%$, muy frecuentemente; y el $34.2 \%$ no lo hace nunca o casi nunca.

El $58.6 \%$ de los docentes nunca o casi nunca utilizan este tipo de aplicaciones como apoyo en sus actividades pedagógicas; el $22 \%$, lo hace ocasionalmente, mientras que el $20 \%$ las utilizan a menudo o muy frecuentemente.

Se logró identificar que en un $34.1 \%$, los docentes hacen uso ocasional de este tipo de aplicaciones para el apoyo de la enseñanza de la geografía; el $49 \%$ las utilizan a menudo o muy frecuentemente; mientras que el $26.9 \%$ nunca o casi nunca las utilizan.

Con relación a esta acción, un $29.3 \%$ de los docentes declaró que ocasionalmente hacen uso de esta herramienta; por su parte, el $51.2 \%$ dijo que nunca o casi nunca la utilizan; y el $19.5 \%$ que frecuente o muy frecuentemente la utilizan.

En este aparte se establece que los docentes, en un $41.4 \%$, hacen uso muy frecuente y frecuentemente de estas aplicaciones, mientras que un $19.5 \%$ lo hacen ocasionalmente, y el $39 \%$ no lo hacen nunca o casi nunca.

Para este criterio, un $75.6 \%$ de los docentes manifestaron hacer uso de esta conexión muy frecuente y frecuentemente, mientras que el $17.1 \%$ lo hace ocasionalmente, y el $7.3 \%$ nunca o casi nunca la utilizan. El $58.6 \%$ lo utilizan muy frecuente o frecuentemente, y el $29.3 \%$, ocasionalmente, mientras que el $12.2 \%$ casi nunca o nunca lo utilizan.

Para este análisis, los docentes expresaron en un $63.4 \%$ que hacen uso de esta herramienta muy frecuente o frecuentemente, el $17.1 \%$, ocasionalmente y el $19.5 \%$, casi nunca o nunca.

Los docentes expresaron que en un $48.8 \%$ hacen uso de esta herramienta muy frecuente 0 frecuentemente; el $46.3 \%$ la utilizan ocasionalmente; mientras que el $4.9 \%$, casi nunca.

Un $95.1 \%$ aseguró que hacen uso muy frecuente o frecuentemente de esta herramienta, y solo el $4.9 \%$ indicó que la utilizan de forma ocasional

Los docentes, en un $17.1 \%$, hacen uso de esta aplicación muy frecuente o frecuentemente; el $17.1 \%$ lo hace ocasionalmente; y el $65 \%$ nunca o casi nunca hace uso de ella.

El $65 \%$ de los encuestados nunca o casi nunca utiliza esta herramienta, el $17.1 \%$ la usa ocasionalmente y el $17.1 \%$ la utilizan muy frecuente o frecuentemente.

El $51.2 \%$ utilizan esta herramienta muy frecuente o frecuentemente, el $26.8 \%$ la utilizan ocasionalmente, y entre el $9.8 \%$ y el $12.2 \%$ no la utilizan nunca o casi nunca.

Los docentes, en un $58.5 \%$, casi nunca o nunca utilizan esta herramienta tecnológica, mientras que el $22 \%$ la utiliza ocasionalmente, y el $19.6 \%$ la usa muy frecuente o frecuentemente.

El $39 \%$ de los encuestados nunca o casi nunca utiliza este tipo de herramientas como apoyo lúdico en los procesos de enseñanza-aprendizaje; por su parte, el $29.3 \%$ respondió que hacen usos de ellas ocasionalmente, y entre el $12.2 \%$ y el $19.5 \%$ las utilizan de forma muy frecuente o frecuentemente.

El $51.3 \%$ nunca o casi nunca utiliza este tipo apoyos tecnológicos para el desarrollo de sus actividades pedagógicas con los estudiantes, lo cual es desaprovechado para el mejoramiento de los procesos de enseñanza-aprendizaje; mientras que el $24.4 \%$ los utiliza de forma ocasional, y el $24.4 \%$ lo hacen de manera frecuente o muy frecuente.

El 58.5 \% hace uso frecuente o muy frecuentemente de esta acción que permite simplificar los procesos por medio de las aplicaciones; el $19.5 \%$ lo hace ocasionalmente; y entre el $12.2 \%$ y el $9.8 \%$ nunca o casi nunca hacen uso de esta opción.

El $61 \%$ de los encuestados las utilizan de forma frecuente o muy frecuente; el $19.5 \%$, ocasionalmente; y el $19.6 \%$ nunca o casi nunca utilizan estas herramientas. 
En la Figura 7 se presentan algunas aplicaciones móviles logradas por los docentes sin experiencia en programación luego de seguir las fases del modelo de desarrollo presentado en este artículo.

En este sentido, las aplicaciones elegidas comparten las siguientes características: 1) son de fácil uso para docentes no programadores; 2) ofrecen contenidos contextualizados a la educación que requieren los participantes estudiantes, a saber, contenidos mayoritariamente de inglés, ciencias, estadística, matemáticas y física; y 3) cuentan con condiciones necesarias para ponerse en práctica al interior de las aulas de clase, esto implicó el análisis de las características de los equipos en cuanto a portabilidad, como lo son las dimensiones de la pantalla y teclado, y las características técnicas, tales como capacidad, velocidad, puertos de conexión y sistemas operativos. Cabe resaltar que dentro de la institución se encuentran equipos diversos que van desde computadores con procesadores de gran velocidad y capacidad, como aquellos que cuentan con las mínimas condiciones para operar. Por lo tanto, la construcción del modelo tuvo como referencia las condiciones técnicas mínimas. Con respecto al segundo punto en mención, se destaca que las categorías que tuvieron un mayor grado de frecuencia con relación a la mediación de aplicaciones móviles en el proceso de enseñanzaaprendizaje fueron: didáctica, aprendizaje y diseño, aspectos que se tuvieron en cuenta para la elección de las aplicaciones expuestas en la Figura 7.
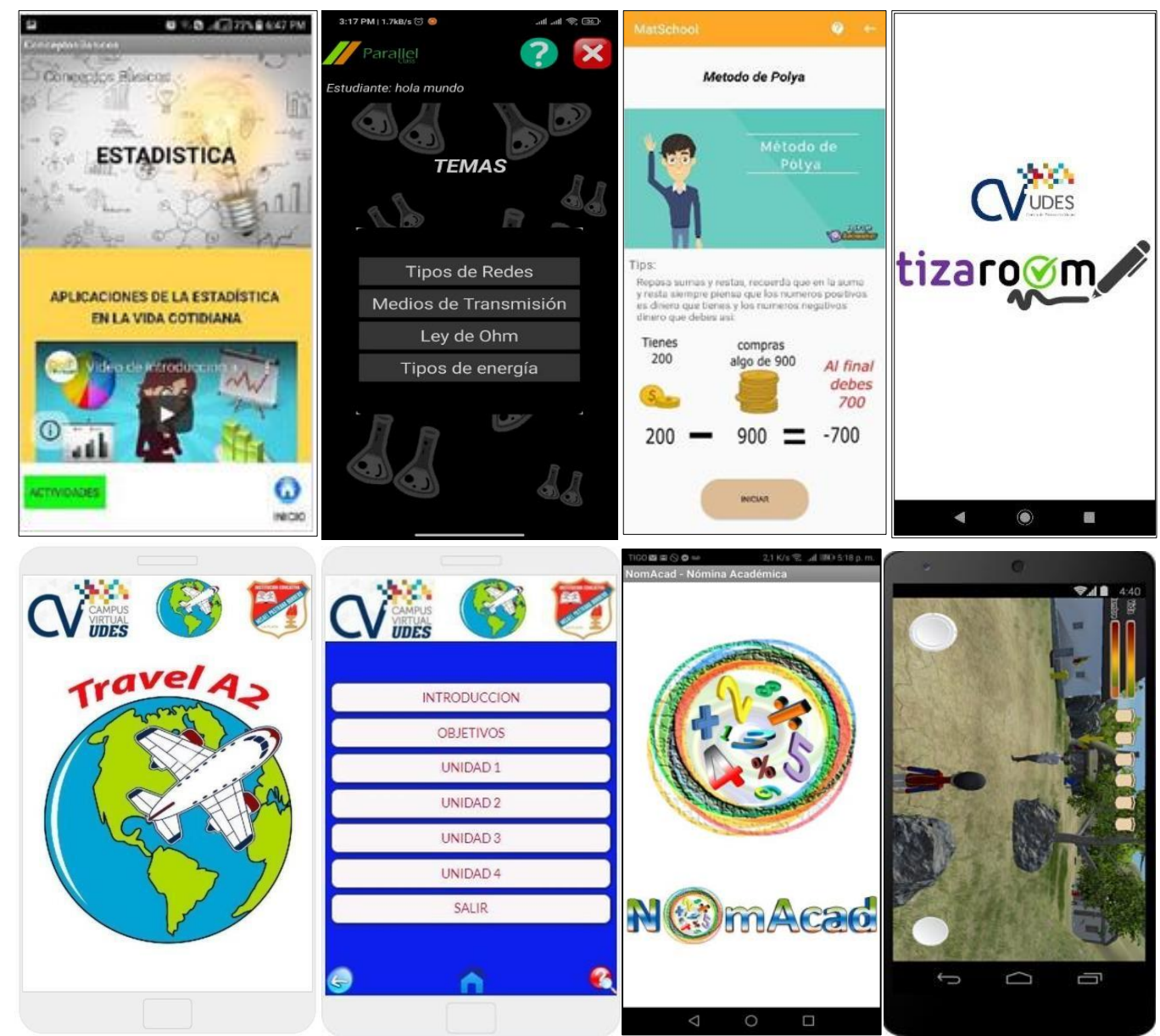

Figura 7. Aplicaciones móviles implementadas con el modelo de desarrollo de aplicaciones móviles educativas Fuente: elaboración propia. 
Por lo anterior, estas aplicaciones dan cuenta de cada una de las etapas descritas en el modelo, para consolidar así, productos de muy buena calidad, y de impacto y significancia para el entorno educativo [65].

A continuación, se explica de forma general el objetivo de cada una de estas aplicaciones:

- Estadística: mejorar las competencias en pensamiento matemático aleatorio en estudiantes de educación media de un contexto rural.

- Travel A2: fortalecer la comprensión de textos en el área de inglés en los estudiantes de sexto grado sexto.

- Parallel: mejorar los resultados valorativos en el área de Tecnología e Informática de los estudiantes de grado noveno.

- $\quad$ NomAcad: fortalecer el proceso de enseñanza-aprendizaje de las competencias contables en grado décimo.

- MatSchool: desarrollar la competencia de resolución de problemas matemáticos que involucren sumas y restas en los estudiantes de grado sexto y séptimo.

- Tizaroom: mejorar la comprensión de las temáticas razones y proporciones del área de Matemáticas en estudiantes de grado séptimo.

- Huellas de Libertad: generar un fortalecimiento de las competencias científico-sociales en el grado quinto.

Bajo el objetivo de implementar el modelo de desarrollo que coadyuve a utilizar estrategias didácticas y metodológicas con la utilización de aplicaciones móviles orientadas al mejoramiento académico, a continuación, se presentan los resultados de la aplicación del instrumento de encuesta de validación a los docentes de las diez instituciones educativas que desarrollaron sus proyectos haciendo uso del modelo de este trabajo bajo el tópico de mediaciones por aplicaciones móviles. Desde el enfoque cualitativo, la validación de este instrumento se realizó a través de juicio de expertos, tomando como referente el método Delphi [66]-[68], con siete doctores especialistas en aprendizaje móvil, con experiencia en aplicaciones móviles para la enseñanza y líderes en procesos tecno-pedagógicos. Se solicitó que evaluaran la claridad y pertinencia de las categorías del instrumento, para lo que estimaron, de acuerdo con un puntaje de 1 a 5 , su grado de satisfacción con el cumplimiento de cada criterio, siendo el número 1 la mínima satisfacción y el número 5 la máxima satisfacción.

De acuerdo con los ítems del cuestionario, en cuanto a los criterios de forma y contenido, se obtuvieron los resultados como se muestra en la Tabla 5 (el promedio general es de $76.8 \%$, lo que indica un coeficiente de competencia bueno para los expertos que validaron el instrumento).

También se solicitó revisar la coherencia entre objetivos y categorías, lo que visibilizó la relación encontrada entre estrategias didácticas y metodológicas por medio de aplicaciones móviles como soporte en lo relacionado con planteamientos teóricos y metodológicos del proceso enseñanza-aprendizaje, y que, finalmente, fueron analizadas e interpretadas con el software Atlas.ti [69], [70]. 
Tabla 5. Estadística del instrumento por juicios de expertos palabras clave Fuente: elaboración propia.

\begin{tabular}{|c|c|c|}
\hline Criterio del instrumento & Ítems & Indicador (\%) \\
\hline \multirow{10}{*}{ Forma } & Claridad & 90 \\
\hline & Pertinencia & 80 \\
\hline & Escala & 90 \\
\hline & Ortografía & 80 \\
\hline & Dificulta en la prueba objetiva & 50 \\
\hline & Presentación & 90 \\
\hline & Extensión & 80 \\
\hline & Tamaño letra & 80 \\
\hline & Orden & 80 \\
\hline & Homogeneidad & 70 \\
\hline \multirow{9}{*}{ Contenido } & Coherencia & 80 \\
\hline & Propósito & 70 \\
\hline & Necesidad de formular estrategias metodológicas basada en TIC & 80 \\
\hline & Conduce a la implementación del modelo & 80 \\
\hline & Novedoso & 90 \\
\hline & Redundancia & 30 \\
\hline & Identifica las variables & 80 \\
\hline & Pertinencia en la información & 80 \\
\hline & Correlación & 80 \\
\hline & TOTAL & 76.8 \\
\hline
\end{tabular}

Es importante exponer las categorías abordadas con sus relaciones y palabras clave como se muestran en la Tabla 6, correspondiente a los resultados de aplicación del instrumento de validación de los proyectos desarrollados por los docentes no programadores.

Es de relevancia plantear que la relación presente entre todas las categorías que se muestran en la Tabla 6 corresponde a la corresponsabilidad que tiene cada uno de los procesos y dinámicas; es decir que, en la creación de estrategias didácticas y metodológicas, existe una interdependencia entre todas las categorías levantadas a través del instrumento de validación. En este caso, no existe una categoría que esté aislada de la otra.

A partir de la Tabla 6 se procede a llevar a cabo un análisis cuantitativo entre la relación y las categorías mostradas anteriormente, tomando de referencia los códigos que poseen cada una de ellas y su índice de coocurrencia en las respuestas registradas en el instrumento de validación de la implementación del modelo por los docentes.

Lo anterior demuestra que, efectivamente, las estrategias implementadas estuvieron encaminadas a la solución de las dificultades o problemas y, a partir de esta, se plantearon objetivos que, gracias al proceso investigativo, lograron un importante fortalecimiento de competencias. 
Tabla 6. Categorías sobre la implementación de estrategias didácticas y metodológicas con sus respectivas palabras clave. Fuente: elaboración propia.

\begin{tabular}{lc}
\hline Categorías & Palabras clave \\
\hline Objetivos & Fortalecer \\
& Diagnosticar \\
Corrientes pedagógicas & Diseñar \\
& Implementar \\
& Evaluar \\
\hline & Conectivismo \\
Mediación por aplicaciones móviles & Aprendizaje significativo \\
& Aprendizaje autónomo \\
\hline & TIC \\
Proceso de enseñanza-aprendizaje & Accesibilidad \\
Didáctica \\
Interactividad \\
Usabilidad \\
Navegación \\
Multimedia \\
Motivación \\
\hline
\end{tabular}

Los participantes de las diez instituciones educativas seleccionadas como muestra de esta investigación, una vez identificaron las dificultades en su contexto educativo, se plantearon un objetivo, y para lograrlo diseñaron una propuesta pedagógica en la cual planearon e implementaron diversas y dinámicas estrategias didácticas, mediadas a través de las aplicaciones móviles para potenciar unas competencias específicas al interior de las diferentes áreas del saber, y con ellas brindar soluciones que arrojaron resultados que permitieron impactar positivamente el entorno educativo. Aunque se manifestaron dificultades de tipo técnico y pedagógico, se mitigaron con dinámicas de trabajo en equipo, apoyadas en el aprendizaje autónomo, el aprendizaje significativo, el conectivismo y el aprendizaje móvil.

A continuación, se exponen en la Figura 8 las derivaciones a manera de coocurrencias de la categoría Avances. 


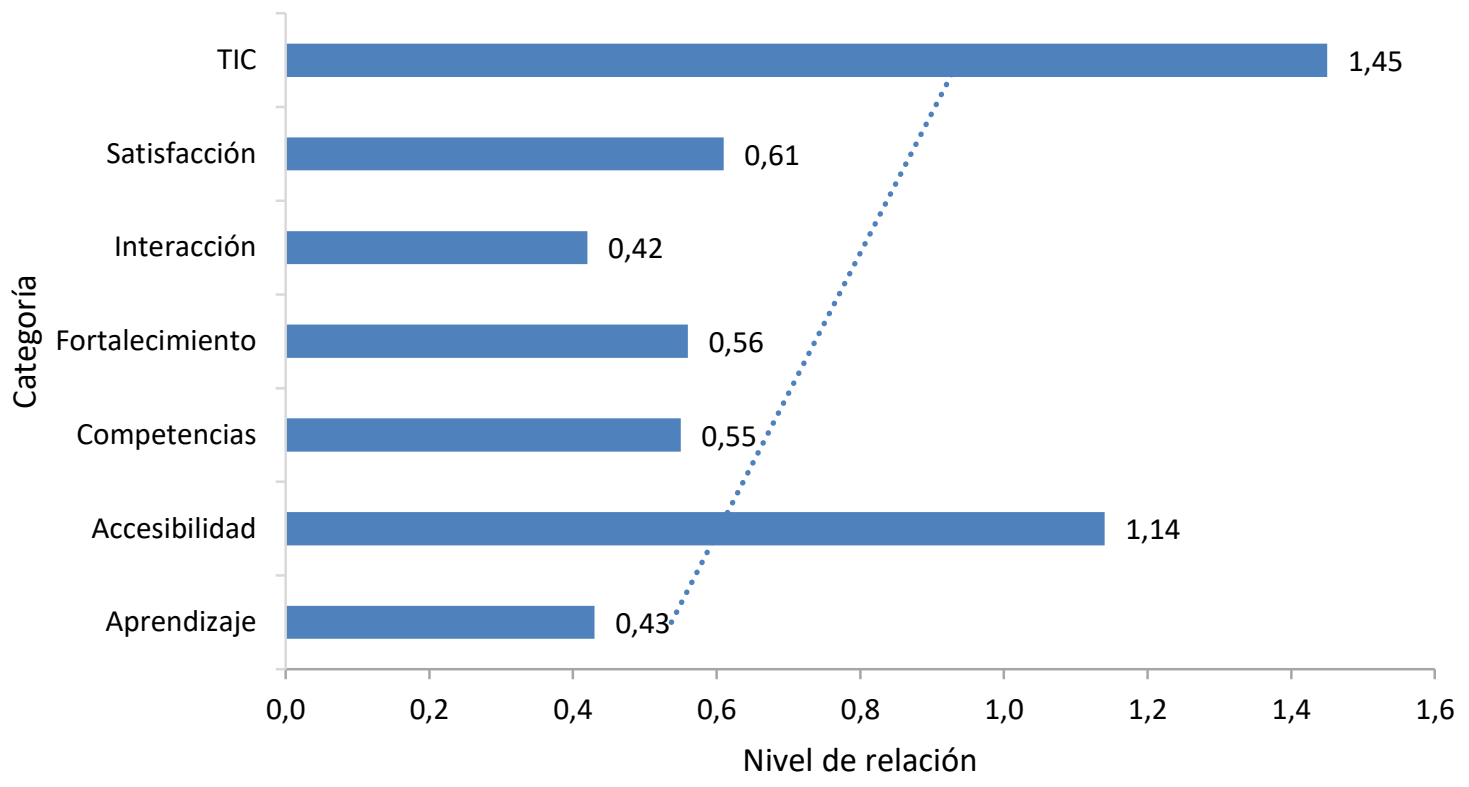

Figura 8. Coocurrencias de avances. Fuente: elaboración propia.

De acuerdo con esto, se presenta un nivel de coocurrencia alto entre la categoría Avances y todas las categorías que posee el documento, lo que la convierte en una de las más relevantes dentro de este análisis.

En la relación establecida desde la categoría Avances, que valora en alto nivel los logros alcanzados por la población objeto de estudio. Esto se materializa al entretejerse con la categoría TIC, la cual se ve reflejada en el más alto valor con $1.45 \%$, en tanto se reconoce como herramienta mediadora para el mejoramiento de los aprendizajes (variable que, a su vez, tiene un $1.14 \%$ ). Con relación al fortalecimiento se reconoce en un $0.56 \%$, lo cual se avala desde una organización didáctica, mediada por unas aplicaciones móviles enlazadas en unas actividades que impactan y cuya satisfacción es reconocida en $0.61 \%$, a través de la interacción, visibilizada en un $0.42 \%$.

Lo anterior, lleva a potenciar unas competencias, representadas en un $0.55 \%$, para lo cual debe apoyarse en recursos que posibiliten una adecuada accesibilidad, reflejada en $0.43 \%$, cubriendo así las expectativas del usuario que se siente cautivado por las TIC, con un valor del $1.45 \%$ para disfrutar de un ambiente participativo, pero que encuentra como dificultad la accesibilidad, con un valor del $0.43 \%$, porque existe un porcentaje alto de los participantes que residen en la zona rural y allí carecen de buena conectividad para el diseño, construcción e implementación de las Aplicaciones Móviles. Sin embargo, los participantes del proyecto crearon, innovaron y le inyectaron estrategias novedosas para cada una de las instituciones educativas, donde desarrollaron cada actividad.

Por otro lado, se tienen como insumos los proyectos de las diez instituciones educativas objeto de estudio de esta investigación, luego de la implementación de las aplicaciones móviles educativas diseñadas y desarrolladas por docentes de diferentes áreas de desempeño. En la Figura 9 se muestran los resultados comparativos por porcentaje de pretest (prueba diagnóstica) y postest (prueba de validación) promediados, al realizar la implementación del modelo de desarrollo de esta investigación, desarrollado por los docentes no programadores en cada institución educativa. 


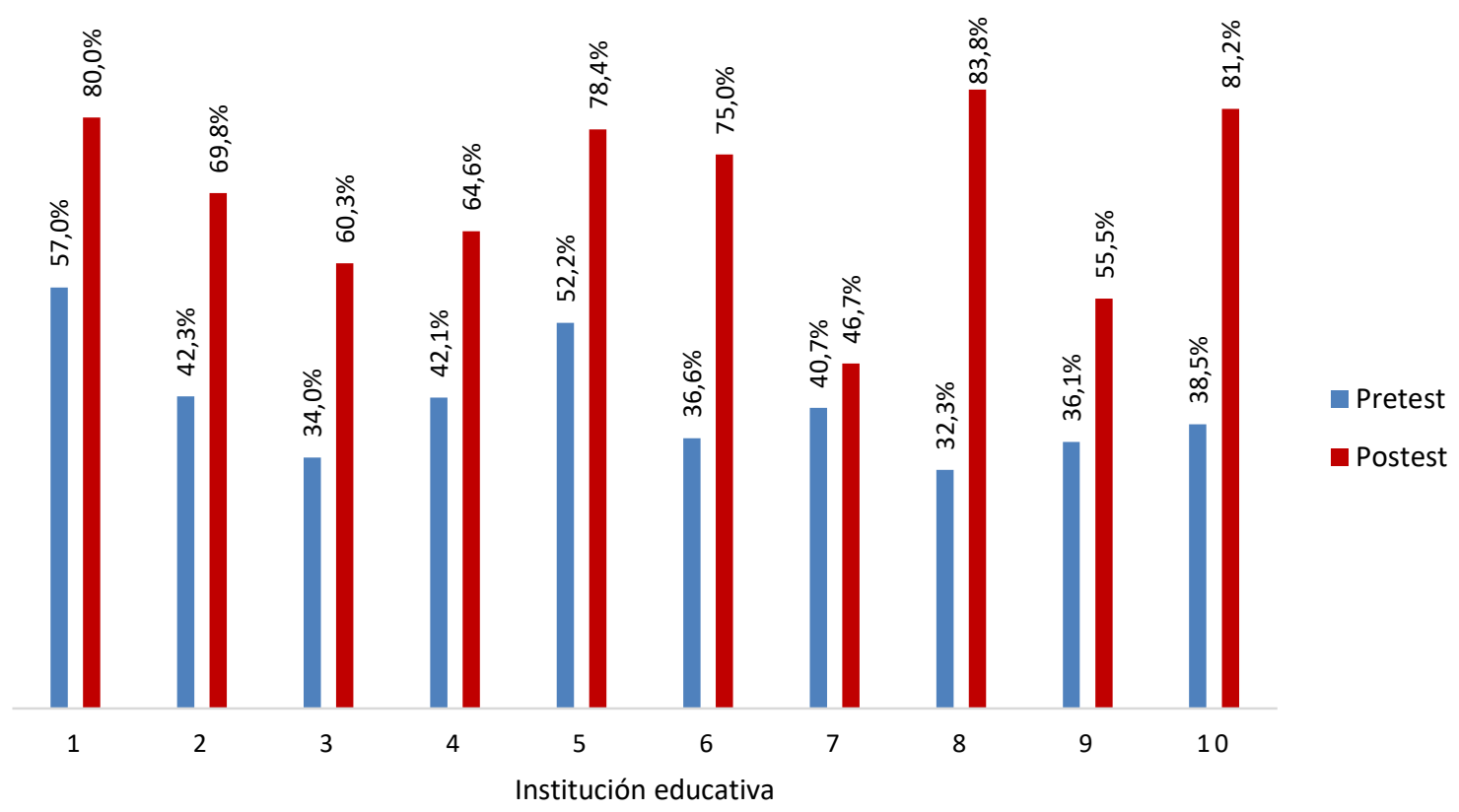

Figura 9. Análisis comparativo de los resultados diagnóstico vs. Resultados de validación Fuente: elaboración propia

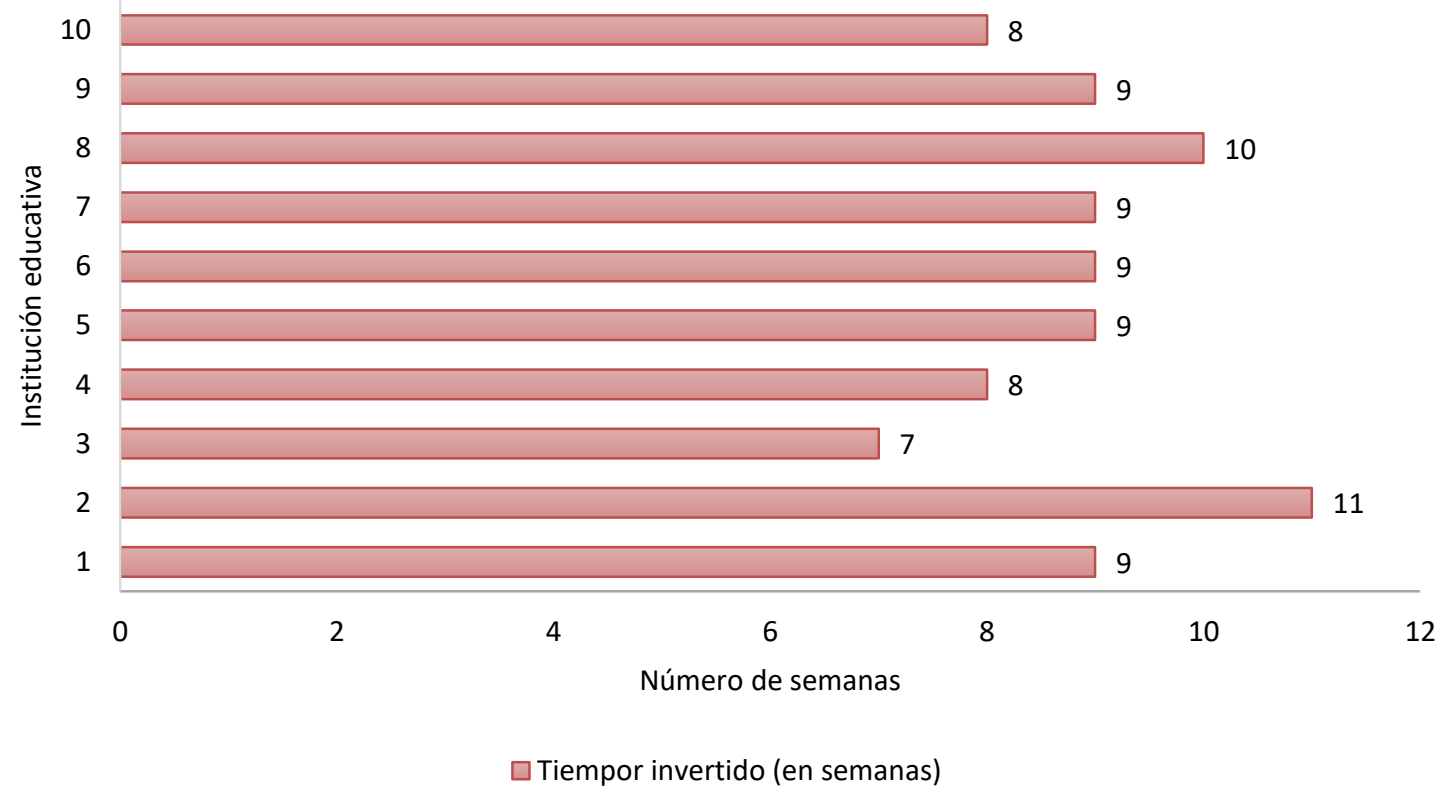

Figura 10. Estadísticas de tiempo de desarrollo de las aplicaciones por institución educativa (IE) Fuente: elaboración propia.

Con relación a la figura anterior:

El objetivo pretest (Figura 9): diagnosticar el nivel de alcance de las competencias de los estudiantes en cada una de las áreas básicas involucradas en el proceso.

El objetivo postest (Figura 9): evaluar los avances en el mejoramiento del aprendizaje de los estudiantes a través de estrategias pedagógicas mediadas por las aplicaciones móviles. 
Cabe aclarar que este pretest y postest no son los mismos a los que se hace referencia en la sección 2.3 relación directa con los resultados expuestos.

A continuación, se presentan en la Figura 10, los tiempos de trabajo representados en números de semanas invertidas por los docentes para el desarrollo de las aplicaciones móviles en cada una de las instituciones educativas involucradas en este estudio. Desde el momento en que se identifica la problemática de aprendizaje en las áreas básicas del saber, los docentes proceden a diseñar la aplicación móvil educativa con la que se realizará la intervención pedagógica. Para ello siguen cada una de las fases del modelo de desarrollo expuesto en este artículo.

Como se evidencia en la Figura 10, el tiempo promedio invertido en el desarrollo de las aplicaciones móviles es de 9 semanas.

\section{DISCUSIÓN}

La evaluación brindada por los expertos (Tabla 5) concluyó que al analizar los instrumentos presentados, estos se encontraban elaborados con claridad, pertinencia y relevancia, dado que de acuerdo con la conceptualización general que se plantea son aplicables para recabar la información requerida en lo pertinente al objeto de estudio [71].

Por otro lado, en los modelos de enseñanza basados en aplicaciones móviles educativas, cabe resaltar que son propuestas que han venido surgiendo a raíz de la necesidad existente de la integración de la m-learning en los procesos de enseñanza y aprendizaje, ya que estas permiten un sinnúmero de ventajas tanto funcionales como pedagógicas [72]. Los modelos de enseñanza aportan distintas ventajas, tales como: facilitar la comunicación y el feedback en el proceso de enseñanza, potenciar la adquisición de los conocimientos y el autoaprendizaje, promover la creatividad e interactividad en los estudiantes y fortalecer el manejo de los recursos tecnológicos que a día de hoy se ha convertido en algo imprescindible para el contexto mundial [34], [73]-[77]. No obstante, el modelo de desarrollo propuesto en este trabajo representa un aporte que coadyuva a que profesores no especializados en desarrollo de software pueden construir aplicaciones móviles educativas para cualquier tipo de plataforma (Android, iOS, entre otras), distinto a los modelos donde se emplean aplicaciones móviles que no son desarrolladas a la medida. De igual manera se reconoce que algunos inconvenientes de este método radican en las características de la portabilidad de los dispositivos debido a las dimensiones de la pantalla y teclado, a lo que se suman los costos que implica un equipo con capacidades óptimas en calidad de características internas, tales como la capacidad y velocidad.

Se encuentra en los resultados una relación directa entre los objetivos y las palabras clave: fortalecer, diagnosticar, diseñar, implementar y evaluar, las cuales se convierten en procesos utilizados por el grupo de participantes en el momento de implementar estrategias didácticas y metodológicas con la utilización de aplicaciones móviles (ver Tabla 6). Como corrientes pedagógicas se encuentran que las más utilizadas corresponden al conectivismo, aprendizaje autónomo y significativo. El primero en mención está estrechamente relacionado con el aprendizaje para la era digital [78]-[80], esto quiere decir que dentro de la configuración del nuevo escenario de enseñanza-aprendizaje la tecnología juega un rol significativo. Es preciso mencionar aquí que, en la educación mediada por las tecnologías, es necesario la búsqueda de estrategias didácticas [81] o modelo de enseñanza que permitan una implicación del estudiante dentro de su proceso de aprender. Esto último en coherencia con el aprendizaje significativo. 
Todo esto demuestra que, efectivamente, el modelo de desarrollo implementado estuvo encaminado a la solución de las dificultades o problemas y a partir de esta se plantearon objetivos que gracias al proceso investigativo lograron un importante fortalecimiento de competencias.

Los participantes de los proyectos seleccionados como muestra, una vez identificaron las dificultades en su contexto educativo, se plantearon un objetivo, y para lograrlo diseñaron una propuesta pedagógica en la cual planearon e implementaron diversas y dinámicas estrategias didácticas, mediadas a través de las aplicaciones móviles para potenciar unas competencias específicas al interior de las diferentes áreas del saber y con ellas brindar soluciones que arrojaron resultados que permitieron impactar positivamente el entorno educativo. Aunque se manifestaron dificultades de tipo técnico y pedagógico, se mitigaron con dinámicas de trabajo en equipo, apoyadas en el aprendizaje autónomo, aprendizaje significativo, conectivismo y aprendizaje móvil. De este modo, las dificultades de estos estudios radican, en gran medida, en la disponibilidad de equipos e infraestructura tecnológica y en la capacidad de estos.

$\mathrm{Al}$ analizar las medias de la prueba postest en comparación con el pretest (ver Figura 9) en el análisis comparativo de los resultados de diagnóstico vs. los resultados de validación, se evidencia un indiscutible mejoramiento de los grupos experimentales, en donde aplicaron la intervención con la aplicación móvil procedente del modelo de desarrollo de esta investigación por parte de los docentes de las instituciones educativas; lo anterior demuestra que el modelo de desarrollo coadyuva en los procesos enseñanza-aprendizaje, llevando a la intervención pedagógica a obtener mejores resultados en las estrategias tecno-pedagógicas [82] y que, por ende, el modelo de desarrollo objeto de estudio de la presente investigación tribute con mayor aceptación para su adaptación a los modelos de enseñanza de los profesores.

De acuerdo con lo anterior, el modelo de desarrollo de aplicaciones móviles para docentes no programadores presentado en este trabajo aporta conocimiento al campo educativo y técnico. Con relación al primer campo en mención, la discusión sobre el aporte se ha venido desarrollando a lo largo del texto, destacándose el impacto que tuvo con relación a sus resultados. La consideración de las características de los estudiantes y de los docentes no programadores permitió adaptar los componentes tecnológicos a las estrategias pedagógicas y con esto desarrollar una aplicación móvil original para el contexto que impactó.

Con respecto a lo técnico, es importante mencionar que la innovación radica en la adecuación del modelo de desarrollo al contexto colombiano, teniendo en cuenta desafíos e implicaciones técnicos diferentes a otros contextos. La lectura contextual marca una diferenciación en la forma de producir modelos de desarrollo de aplicaciones móviles, pues dependiendo de estas características el equipo técnico desarrolla un modelo que pueda impactar positivamente.

Este interés de conocer las características del contexto y los usuarios para la creación técnica del modelo de la aplicación, se convierte en una estrategia que se comparte con otros estudios y que permite la investigación y creación de Ciencia, Tecnología e Innovación (CTeI) [83]-[85]. En este sentido, se puede afirmar que cada proceso de aplicación móvil en el campo pedagógico es una innovación que debe adecuarse a la tecnología del contexto y a los usuarios de manera particular. Es así como en países como Grecia [85], China [86], Estados Unidos [87] o Taiwán [88] se reconocen los avances en tecnología y la amplitud de la cobertura de la enseñanza virtual, por esto sus intereses difieren del nuestro en la medida que pretenden generar innovación-creación desde un nivel avanzado, por medio del trabajo de la realidad aumentada, reconocimiento facial y modelación en 3D, entre otros.

En este orden de ideas, de acuerdo con la lectura del contexto, el trabajo permitió un modelo de desarrollo orientado a la construcción de aplicaciones móviles educativas para 
docentes no desarrolladores que puedan ponerse en práctica al interior de las aulas de clase, generando procesos de capacitación a los docentes para que tengan la posibilidad de diseñar y desarrollar sus propias aplicaciones y así contribuir con el mejoramiento académico de sus estudiantes. Por lo tanto, las indagaciones del contexto de la fase diagnóstica permitieron plantear el modelo de desarrollo teniendo en cuenta la alfabetización tecnológica que se requería para estudiantes y docentes, como también las condiciones de accesibilidad.

En este sentido, la originalidad y el valor que cobra este estudio radica en la posibilidad de crear el desarrollo de la aplicación móvil de manera contextualizada a las características tecnológicas y educativas del país, pues, aunque se tuvo como referente los desarrollos en aplicaciones móviles de diferentes países del mundo, el equipo de investigación admitió las limitaciones en la accesibilidad del contexto y, finalmente, fue esto lo que permitió la originalidad del trabajo. Es decir, las limitaciones del método radican en las mismas del contexto tecnológico en donde se encuentran los equipos.

\section{CONCLUSIONES}

La tendencia actual en el uso de dispositivos móviles en la educación se centra en el hecho de que, en el futuro, estos dispositivos se utilizarán cada vez más en las aulas y centros educativos y culturales. Estos dispositivos fueron originalmente diseñados para la comunicación, y su aparición ha provocado un cambio de paradigma en la educación general.

El uso de dispositivos móviles en la educación es un elemento básico de la construcción del conocimiento, pues a través de la utilización de estas tecnologías se incrementa la posibilidad de interactuar con los miembros del grupo, mejorando así la comunicación.

El modelo de desarrollo de este estudio permite a los docentes que no son desarrolladores desplegar aplicaciones educativas en dispositivos móviles que sirven como herramienta didáctica en el proceso de enseñanza y aprendizaje de la educación primaria y secundaria básica.

Para el caso de esta investigación, el uso de App Inventor y el uso de Wysiwyg permite que la construcción de recursos educativos digitales no sea tarea exclusiva de los expertos en desarrollo de software. Esto en la medida que este trabajo promueve que el docente con experticia en pedagogía se involucre en tareas de arquitectura de la información (sketch, wireframes y mockup) y desarrollo de software. En la representación de la muestra de los proyectos que se desarrollaron, con el objetivo de implementar un modelo de desarrollo de aplicaciones móviles educativas a la medida y orientadas al mejoramiento académico, se observa un sistema conformado por cinco categorías (Tabla 6) con una marcada relación con sus respectivos códigos. La categoría proceso de enseñanza-aprendizaje, desde sus códigos es permeada a través de las demás categorías como: objetivos, corrientes pedagógicas, resultado, mediaciones por aplicaciones móviles, enfoque de investigación y dificultades. Este entramado de relaciones propició un escenario educativo interactivo y dinámico, fortalecido desde unos parámetros de innovación tecnológica que, a través de estrategias didácticas, medió en aras de potenciar una escuela con avances hacia un escenario tecno-pedagógico.

En este sentido, la mediación de aplicaciones móviles de este tipo facilita el proceso académico de las áreas de desempeño mediante una buena planificación de actividades y secuencias didácticas como se propone en este modelo de desarrollo. Al usar aplicaciones diseñadas para ser navegables, accesibles, utilizables, interactivas, estimulantes y multimediales, además, de la pedagogía integrada por medio de esta TIC, se puede mejorar la capacidad de desarrollo, fortaleciendo así el nivel de aprendizaje de los estudiantes y potenciando su poder de educación técnica. La fuerza tecno-pedagógica, enmarcada en sus 
códigos de fortalecimientos, aprendizaje, secuencia didáctica, conclusiones, TIC y didáctica, establece los criterios para que las aplicaciones móviles se conviertan en herramientas de aprendizaje entretenidas y útiles, aprovechando el componente visual como su máximo atractivo y empleando diversos recursos multimediales.

Sumado a lo anterior, el uso de la App Inventor y Wysiwyg dinamiza los entornos tecnopedagógicos en los escenarios educativos en los cuales se lidera la apropiación de los recursos como mediadores en los procesos de enseñanza-aprendizaje, con la pretensión de desarrollar actividades de formación, capacitación y actualización para la comunidad educativa y potenciar el fortalecimiento de las habilidades, competencias y desempeños de los estudiantes en los diferentes contenidos y componentes de todas y cada una de las áreas del conocimiento.

Por lo tanto, las aplicaciones móviles educativas, en especial la abordada en este estudio, son utilizadas como herramientas mediadoras de aprendizaje que posibilitan transversalizar desde cada una de las áreas del saber, permean estilos y prácticas pedagógicas, transforman escenarios educativos en ambientes de aprendizaje participativos, lúdicos, cautivadores de atención y garantes de permanencia en las instituciones, dado que se fortalece el robustecimiento de calidad en la prestación del servicio educativo.

Con relación a los trabajos futuros, se considera pertinente el seguimiento de este estudio para conocer, de manera longitudinal, la sostenibilidad en el tiempo de esta aplicación móvil, y también sus fortalezas e inconvenientes para consolidar ajustes que permitan optimizarla y contextualizarla a las transformaciones en las dinámicas educativas. De igual manera se propone a la comunidad académica la promoción del uso y desarrollo de aplicaciones móviles para su implementación en espacios educativos debido a los resultados óptimos en el impacto de la enseñanza-aprendizaje presentados en este estudio, y a la comunidad científica la realización de investigaciones como esta que permitan dar soporte teórico a este tipo de enseñanzas.

\section{AGRADECIMIENTOS}

Este trabajo se desarrolló bajo el proyecto de investigación "Diseño y desarrollo de aplicaciones móviles para el mejoramiento del proceso enseñanza aprendizaje en las instituciones educativas públicas del país", financiado por la convocatoria para proyectos de investigación, desarrollo tecnológico, innovación o creación VII-FT-026- Universidad de Santander (UDES), 2020. Apoyado, además, por la Universidad Cooperativa de Colombia (Montería - Colombia), la Universidad Internacional Iberoamericana (Campeche - México), la Universidad de Córdoba (Montería - Colombia) y las diez instituciones educativas participantes en el proceso investigativo.

\section{CONFLICTO DE INTERESES}

Los autores declaran no tener ningún conflicto de interés.

\section{CONTRIBUCIÓN DE LOS AUTORES}

J. Escobar-Reynel: conceptualización, metodología, software, investigación, redacciónelaboración del borrador original, investigación, validación, concibió la idea original, edición, autor corresponsal. 
R. Baena-Navarro: metodología, curación de datos, redacción-elaboración del borrador original, investigación, validación.

B. Giraldo-Tobón: metodología, adquisición de datos, redacción-elaboración del borrador original, investigación.

M. Macea-Anaya: software, adquisición de datos, validación, supervisión, revisión de escritura.

S. Castaño-Rivera: software, validación, revisión de escritura, edición.

\section{REFERENCIAS}

[1] C. J. de Brabander; F. J. Glastra, "The unified model of task-specific motivation and teachers' motivation to learn about teaching and learning supportive modes of ICT use”, Educ. Inf. Technol., vol. 26, no. 1, pp. 393-420, Jan. 2021. https://doi.org/10.1007/s10639-020-10256-7

[2] R. Rueda Ortiz; M. Franco-Avellaneda, "Políticas educativas de TIC en Colombia: entre la inclusión digital y formas de resistencia-transformación social”, Pedagog. y Saberes, no. 48, pp. 9-25, Jan. 2018. https://doi.org/10.17227/pys.num48-7370

[3] R. A. Builes Múnera, "El aporte de Computadores para Educar en la implementación de la Enseñanza Innovadora”, Rev. Virtual Univ. Católica del Norte, vol. 44, pp. 291-303, Feb. 2015. URL

[4] L. Sierra Peñuela, "Maestros, Apropiación De TICs Y Desempeño Escolar En Colombia”, Documentos CEDE, vol. 56, pp. 1-40, Oct. 2017. http://dx.doi.org/10.2139/ssrn.3047310

[5] M. Alzate, "Reconciliación social: tecnología para la construcción de paz", TecnoLógicas, vol. 20, no. 39, pp. 9-13, May 2017. https://doi.org/10.22430/22565337.739

[6] Colciencias, "Adenda 003", 2014. URL

[7] Departamento Nacional de Planeación; Ministerio de Educación Nacional; Ministerio de las Tecnologías de la Información y las Comunicaciones, "Documento CONPES 3988. Tecnologías para aprender: Política nacional para impulsar la innovación en las prácticas educativas a través de las tecnologías digitales”, 2020. $\underline{\mathrm{URL}}$

[8] L. E. Molina-Pacheco; F. Y. Mesa-Jiménez, "Las TIC en escuelas rurales: realidades y proyección para la integración", Prax. Saber, vol. 9, no. 21, pp. 75-98, Sep. 2018.

https://doi.org/10.19053/22160159.v9.n21.2018.8924

[9] J. P. Meneses Ortegón; T. Jové; J. Puiggalí; R. Fabregat, "Representación del conocimiento de un proceso de co-creación de material educativo", TecnoLógicas, vol. 23, no. 47, pp. 159-177, Jan. 2020.

http://dx.doi.org/10.22430/22565337.1493

[10] N. D. Duque Méndez; P. A. Rodríguez Marín; C. A. Collazos, "Informática en la Educación”, TecnoLógicas, vol. 23, no. 47, pp. 1-2, Jan. 2020. https://doi.org/10.22430/22565337.1569

[11] J. F. Restrepo Tamayo; D. S. Gómez Agudelo, "Digital Connectivity as a Fundamental Right in Colombia", Law, State Telecommun. Rev., vol. 12, no. 1, pp. 113-136, Apr. 2020. https://doi.org/10.26512/lstr.v12i1.31161

[12] Ministerio de Tecnologías de la información y las comunicaciones, "Plan Nacional de Conectividad Rural", Colombia, 2019. URL

[13] M. Palacio Puerta; K. I. Cabrera Peña, "La gobernanza de internet como plataforma para impulsar políticas en la educación con TIC. El caso de Colombia”, OPERA, no. 21, pp. 5-23, Nov. 2017. http://dx.doi.org/10.18601/16578651.n21.02

[14] C. Ricardo; M. Borjas; I. Velásquez; J. Colmenares; A. Serje, "Caracterización de la integración de las TIC en los currículos escolares de instituciones educativas en Barranquilla”, Zo. Próxima, no. 18, pp. 32-45, Jan. 2013. URL

[15] F. A. Poveda Aguja, "Reflexión, acción y transformación en la enseñanza-aprendizaje en docentes y estudiantes de las instituciones educativas beneficiarias del Programa Computadores para Educar con la inclusión de las tecnologías de la información y comunicación”, Rev. Virtual Univ. Católica del Norte, no. 43, pp. 161-179, Oct. 2014. URL

[16] J. A. Cortés Barrios, "Eficacia del Programa Computadores Para Educar en la Apropiación de las TIC en los Estudiantes de Media de los Colegios Oficiales Nueva Delhi y La Belleza”. (Tesis de Maestría), Universidad Externado de Colombia, Bogotá. 2018. URL

[17] V. Jordán; H. Galperin; W. Perez Núñez, "Banda ancha en América Latina: más allá de la conectividad", Naciones Unidas - CEPAL, 2013. URL

[18] E. Said Hung et al., "Hacia el Fomento de las TIC en el Sector Educativo en Colombia", Barranquilla, 
Colombia: Ediciones Uninorte, 2015.URL

[19] N. Refat; H. Kassim; M. A. Rahman; R. Bin Razali, "Measuring student motivation on the use of a mobile assisted grammar learning tool”, PLoS One, vol. 15, no. 8, e0236862, Aug. 2020. https://doi.org/10.1371/journal.pone.0236862

[20] Y.-S. Chang; K.-J. Hu; C.-W. Chiang; A. Lugmayr, "Applying Mobile Augmented Reality (AR) to Teach Interior Design Students in Layout Plans: Evaluation of Learning Effectiveness Based on the ARCS Model of Learning Motivation Theory", Sensors, vol. 20, no. 1, p. 105, Dec. 2019. https://doi.org/10.3390/s20010105

[21] D. Ragupathi; N. Ibrahim; K.-A. Tan; B. N. Andrew, "Relations of Bedtime Mobile Phone Use to Cognitive Functioning, Academic Performance, and Sleep Quality in Undergraduate Students", Int. J. Environ. Res. Public Health, vol. 17, no. 19, pp. 7131, Sep. 2020. https://doi.org/10.3390/ijerph17197131

[22] F. Trede; L. Markauskaite; C. McEwen; S. Macfarlane, Education for Practice in a Hybrid Space, Singapore: Springer, 2019.

[23] M. D. Bustamante-Rodríguez; A. A. Piedrahita-Ospina; I. M. Ramírez-Velásquez, "Modelo para detección automática de errores léxico-sintácticos en textos escritos en español", TecnoLógicas, vol. 21, no. 42, pp. 199-209, May. 2018. https://doi.org/10.22430/22565337.788

[24] R. Zamora Delgado, "El M-Learning, las ventajas de la utilización de dispositivos móviles en el proceso autónomo de aprendizaje", Rehuso, vol. 4, no. 3, pp. 29-38, Sep. 2019. URL

[25] C. M. Stewart; C. C. Schifter; M. E. Markaridian Selverian, Teaching and Learning with Technology, New York: Taylor \& Francis Group, 2010.

[26] C. De Witt; C. Gloerfeld, Handbuch Mobile Learning, Wiesbaden: Springer Fachmedien Wiesbaden, 2018.

[27] B. Gros Salvat, "La evolución del e-learning: del aula virtual a la red", RIED. Rev. Iberoam. Educ. a Distancia, vol. 21, no. 2, pp. 69-82, Jul. 2018. https://doi.org/10.5944/ried.21.2.20577

[28] L. M. Pilotto, Blended Learning, Wiesbaden: Springer Fachmedien Wiesbaden, 2021. https://doi.org/10.1007/978-3-658-10175-6

[29] N. J. van Eck; L. Waltman, "VOS: A New Method for Visualizing Similarities Between Objects", in Advances in Data Analysis. Studies in Classification, Data Analysis, and Knowledge Organization, Springer, Berlin, Heidelberg, 2007, pp. 299-306. https://doi.org/10.1007/978-3-54

[30] D. Galin, Software quality: concepts and practice. Wiley-IEEE Press, 2018. URL

[31] P. Markopoulos; B. De Ruyter; W. Mackay, "Awareness Systems Advances in Theory, Methodology and Design” in Human-Computer Interaction Series, London: Springer, 2009. https://doi.org/10.1007/978-184882-477-5

[32] R. Hernández Sampieri; C. P. Mendoza Torres, Metodología de la investigación. Las rutas cuantitativa, cualitativa y mixta, Ciudad de México: Mc Graw Hill, 2018

[33] J. Arias-Gómez; M. Á. Villasís-Keever; M. G. Miranda Novales, "El protocolo de investigación III: la población de estudio", Revista Alergia México, vol. 63, no. 2, pp. 201-206, May. 2016. https://doi.org/10.29262/ram.v63i2.181

[34] S. Gómez-Ardila; A. Chacón-González, "Aprendizaje móvil basado en el modelo Frame y aplicado al aprendizaje de la técnica de Core en Fisioterapia”, Rev. Virtual Univ. Católica del Norte, no. 50, pp. 411436, Mar. 2017. URL

[35] J. M. Molina; D. Romero, "Mobile Learning Environment based on Micro-Learning", Rev. Iberoam. Tecnol. del Aprendiz., vol. 5, no. 4, pp. 159-166, Nov. 2010. URL

[36] A. M. Antelm Lanzat; A. J. Gil López; M. L. Cacheiro González; E. Pérez Navío, "Causas del fracaso escolar: Un análisis desde la perspectiva del profesorado y del alumnado", Enseñanza Teach. Rev. Interuniv. Didáctica, vol. 36, no. 1, pp. 129- 149, Jun. 2018. https://doi.org/10.14201/et2018361129149

[37] O. Y. Aparicio Gómez, "Uso y apropiación de las TIC en educación", Rev. Interam. Investig. Educ. y Pedagog. RIIEP, vol. 12, no. 1, pp. 253-284, Jan. 2019. https://doi.org/10.15332/s1657-107X.2019.0001.04

[38] L. Collins; S. R. Ellis, Mobile Devices: Tools and Technologies, New York: Chapman and Hall/CRC, 2015.

[39] F. Kamriani; K. Roy, App Inventor 2 Essentials, EE.UU, Packt Publishing, 2016.

[40] H. T. G. Smith, Software Quality Assurance, A Guide for Developers and Auditors, Florida: CRC Press, 2020.

[41] D. Churchill; B. Fox; M. King, Framework for Designing Mobile Learning Environments. Singapore: Springer Singapore, pp. 3-25, 2016. https://doi.org/10.1007/978-981-10-0027-0_1

[42] J. Keengwe, Handbook of Research on Digital Content, Mobile Learning, and Technology Integration Models in Teacher Education. IGI Global, 2018.

[43] D. Travis; P. Hodgson, Think Like a UX Researcher: How to Observe Users, Influence Design, and Shape Business Strategy. CRC Press, 2019.

[44] J. Yablonski, Laws of UX: Design Principles for Persuasive and Ethical Products. O'Reilly Media, 2020.

[45] P. B. Deacon, UX and UI Strategy: A step by step Guide on UX and UI design, EE.UU.: Independently published, 2020. 
[46] J. Ligman; M. Pistoia; O. Tripp; G. Thomas, "Improving design validation of mobile application user interface implementation", in Proceedings of the International Conference on Mobile Software Engineering and Systems, May. 2016, pp. 277-278. https://doi.org/10.1145/2897073.2897708

[47] S. Barra; R. Francese; M. Risi, "Automating Mockup-Based Usability Testing on the Mobile Device", en International Conference on Green, Pervasive, and Cloud Computing GPC 2019, Suiza, 2019. pp. 128-143. https://doi.org/10.1007/978-3-030-19223-5 10

[48] S.-Ch. Kong; H. Abelson, Computational Thinking Education, Singapore: Springer, 2019. URL

[49] J. Landauer; M. Hirakawa, "From Programming by Demonstration to Programming by WYSIWYG", J. Vis. Lang. Comput., vol. 8, no. 5-6, pp. 621-640, Dec. 1997. https://doi.org/10.1006/jvlc.1997.0063

[50] Y. D. Amaya Balaguera, "Metodologías ágiles en el desarrollo de aplicaciones para dispositivos móviles. Estado actual", Rev. Tecnol., vol. 12, no. 2, pp. 111-124, Dec. 2015.https://doi.org/10.18270/rt.v12i2.1291

[51] I. García Pacheco; J. García Matías, "Una Metodología Basada en Prácticas Efectivas para Desarrollar Software Educativo", Comput. y Sist., vol. 11, no. 4, pp. 313-322, Dec. 2007. URL

[52] Z. Cataldi; F. Lage; R. Pessacq; R. García-Martínez, "Metodología extendida para la creación de software educativo desde una visión integradora”, Rev. Latinoam. Tecnol. Educ., vol. 2, no. 1, pp. 9-40, Jen. 2007. URL

[53] D. Wolber; H. Abelson; E. Spertus; L. Looney, App Inventor 2: Create Your Own Android Apps, 2da ed., O'Reilly Media, Inc., 2014.

[54] I. Ruiz-Rube; J. M. Mota; T. Person; J. M. Rodríguez Corral; J. M. Dodero, "Block-Based Development of Mobile Learning Experiences for the Internet of Things,” Sensors, vol. 19, no. 24, p. 5467, Dec. 2019. https://doi.org/10.3390/s19245467

[55] E. W. Patton, MIT App Inventor for iOS on the App Store, Massachusetts Institute of Technology, 2021.

[56] T. Susi; M. Johannesson; P. Backlund, "Serious Games - An Overview", Univ. Skövde, Sch. Humanit. Informatics., pp. 1-28, 2007. URL

[57] L. Cayola; J. A. Macías, "Systematic guidance on usability methods in user-centered software development", Inf. Softw. Technol., vol. 97, pp. 163-175, May. 2018. https://doi.org/10.1016/j.infsof.2018.01.010

[58] K. Curcio; R. Santana; S. Reinehr; A. Malucelli, "Usability in agile software development: A tertiary study", Comput. Stand. Interfaces, vol. 64, pp. 61-77, May. 2019. https://doi.org/10.1016/j.csi.2018.12.003

[59] A. Kaya; R. Ozturk; C. Altin Gumussoy, Usability Measurement of Mobile Applications with System Usability Scale (SUS), en Industrial Engineering in the Big Data Era, Suiza: Springer, 2019, pp. 389-400. https://doi.org/10.1007/978-3-030-03317-0_32

[60] P. W. Jordan, An Introduction to Usability, London: CRC Press, Aug. 1998.

[61] J. M. Bruel; M. Mazzara; B. Meyer, Software Engineering Aspects of Continuous Development and New Paradigms of Software Production and Deployment, France, Cham: Springer International Publishing, 2019.

[62] L. J. Cronbach, "Coefficient alpha and the internal structure of tests", Psychometrika, vol. 16, no. 3, pp. 297-334, Sep. 1951. https://doi.org/10.1007/BF02310555

[63] J. González; M. Pazmiño, "Cálculo e interpretación del Alfa de Cronbach para el caso de validación de la consistencia interna de un cuestionario, con dos posibles escalas tipo Likert”, Rev. Publicando, vol. 2, no. 2, pp. 62-77, Jan. 2015. URL

[64] R. Ledesma, "AlphaCI: un programa de cálculo de intervalos de confianza para el coeficiente alfa de Cronbach", Psico-USF, vol. 9, no. 1, pp. 31-37, Jun. 2004. https://doi.org/10.1590/S141382712004000100005

[65] M. Hertzum; R. Molich; N. Jacobsen, "What you get is what you see: revisiting the evaluator effect in usability tests", Behav. Inf. Technol., vol. 33, no. 2, pp. 144-162, Apr. 2013. https://doi.org/10.1080/0144929X.2013.783114

[66] C. E. George Reyes; L. Trujillo Liñán, "Aplicación del Método Delphi Modificado para la Validación de un Cuestionario de Incorporación de las TIC en la Práctica Docente”, Rev. Iberoam. Evaluación Educ., vol. 11, no. 1, pp. 113-135, Feb. 2018. https://doi.org/10.15366/riee2018.11.1.007

[67] J. Cabero Almenara; A. Infante Moro, "Empleo del método Delphi y su empleo en la investigación en comunicación y educación”, Edutec. Rev. Electrónica Tecnol. Educ., vol. 48, no. 48, pp. 1-16, Jun. 2014. https://doi.org/10.21556/edutec.2014.48.187

[68] A. M. Soriano Rodríguez, "Diseño y validación de instrumentos de medición”, Diá-logos, no. 14, pp. 19-40, Nov. 2015. https://doi.org/10.5377/dialogos.v0i14.2202

[69] D. San Martín Cantero, "Teoría fundamentada y Atlas.ti: recursos metodológicos para la investigación educativa", Rev. electrónica Investig. Educ., vol. 16, no. 1, pp. 104-122, 2014. URL

[70] C. Penalva-Verdú; A. Alaminos-Chica; F. Francés-García; Ó. Santacreu-Fernández, La investigación cualitativa: técnicas de investigación y análisis con Atlas.ti, Ecuador: PYDLOS Ediciones, 2015.

[71] J. J. Gutiérrez Castillo; J. Cabero Almenara; L. I. Estrada Vidal, "Diseño y validación de un instrumento de evaluación de la competencia digital del estudiante universitario”, Rev. Espac., vol. 38, no. 10, 2017. URL 
[72] C. Villalonga Gómez; C. Marta Lazo, "Modelo de integración educomunicativa de 'apps' móviles para la enseñanza y aprendizaje”, Píxel-Bit, Rev. Medios y Educ., no. 46, pp. 137-153, Jan. 2015. https://doi.org/10.12795/pixelbit.2015.i46.09

[73] P. Bitrián; I. Buil; S. Catalán, "Enhancing user engagement: The role of gamification in mobile apps", J. Bus. Res., vol. 132, pp. 170-185, Aug. 2021. https://doi.org/10.1016/j.jbusres.2021.04.028

[74] S. M. Schueller; C. M. Armstrong; M. Neary; R. P. Ciulla, "An Introduction to Core Competencies for the Use of Mobile Apps in Cognitive and Behavioral Practice", Cogn. Behav. Pract., Jan. 2021. https://doi.org/10.1016/i.cbpra.2020.11.002

[75] S. Martín et al., "M2Learn: Framework abierto para el desarrollo de aplicaciones para el aprendizaje móvil y ubicuo", Rev. Iberoam. Tecnol. del Aprendiz., vol. 5, no. 4, pp. 138-145, Nov. 2010. URL

[76] M. L. Koole, "A model for framing mobile learning", en Mobile Learning: Transforming the Delivery of Education and Training, Canadá, Mohamed Ally, 2009, pp. 25-47. URL

[77] J. M. Molina; D. Romero, "Ambiente de aprendizaje móvil basado en micro-aprendizaje", Rev. Iberoam. Tecnol. del Aprendiz., vol. 5, no. 4, pp. 159-166, Nov. 2010. URL

[78] R. Sanchez-Cabrero; Ó. Costa-Román; L. Mañoso-Pacheco; M. Á. Novillo-López; F. J. Pericacho-Gómez, "Orígenes del conectivismo como nuevo paradigma del aprendizaje en la era digital", Educ. y Humanismo, vol. 21, no. 36, pp. 113-136, Jan. 2019. https://doi.org/10.17081/eduhum.21.36.3265

[79] C. M. Tinoco Díaz; E. K. Tinoco Díaz, "Uso y consumo de las aplicaciones móviles en el Smartphone como herramienta de apoyo académico”, Espacios, vol. 39, no. 30, pp. 18, Apr. 2018. URL

[80] J. M. Traxler, "Learning with Mobiles in Developing Countries: Technology, Language, and Literacy", Int. J. Mob. Blended Learn., vol. 9, no. 2, pp. 1-15, Apr. 2017. https://doi.org/10.4018/IJMBL.2017040101

[81] F. J. Luque Rodríguez, "Las TIC en educación: caminando hacia las TAC", 3C TIC Cuad. Desarro. Apl. a las TIC, vol. 5, no. 4, pp. 55-62, Dec. 2016. https://doi.org/10.17993/3ctic.2016.54.55-62

[82] W. E. Mercado Borja; G. Guarnieri; G. Luján Rodríguez, "Análisis y evaluación de procesos de interactividad en entornos virtuales de aprendizaje”, Trilogía Cienc. Tecnol. Soc., vol. 11, no. 20, pp. 63-99, Jan. 2019. https://doi.org/10.22430/21457778.1213

[83] J. VD. Wirjawan; D. Pratama; E. Pratidhina; A. Wijaya; B. Untung; H. Herwinarso, "Development of Smartphone App as Media to Learn Impulse-Momentum Topics for High School Students", Int. J. Instr., vol. 13, no. 3, pp. 17-30, Jul. 2020. https://doi.org/10.29333/iji.2020.1332a

[84] D. D. M. Dolawattha; S. Pramadasa; P. M. Jayaweera, "Mobile Learning: Modelling the Influencing Factors on Mobile Devices", in 2020 20th International Conference on Advances in ICT for Emerging Regions (ICTer), Colombo. 2020, pp. 1-2. https://doi.org/10.1109/ICTer51097.2020.9325494

[85] C. Pasalidou; N. Fachantidis, "Teachers' Perceptions Towards the Use of Mobile Augmented Reality", en Internet of Things, Infrastructures and Mobile Applications, Suiza: Springer, 2021, pp. 1039-1050. https://doi.org/10.1007/978-3-030-49932-7_97

[86] J. Xiao; E. Tan; X. Li; M. Specht; M. Cao, "Using social media in mobile MOOC for teacher professional development", Int. J. Mob. Learn. Organ., vol. 14, no. 4, p. 492, Jan. 2020. https://doi.org/10.1504/IJMLO.2020.10030690

[87] M. I. Ul Haque; D. Valles, "Facial Expression Recognition Using DCNN and Development of an iOS App for Children with ASD to Enhance Communication Abilities", in 2019 IEEE 10th Annual Ubiquitous Computing, Electronics \& Mobile Communication Conference (UEMCON), New York. 2019, pp. 0476-0482. https://doi.org/10.1109/UEMCON47517.2019.8993051

[88] W.-H. Chao; C.-Y. Yang; R.-C. Chang, "A Study of the Interactive Mathematics Mobile Application Development”, in 2018 1st IEEE International Conference on Knowledge Innovation and Invention (ICKII), Jul. 2018, pp. 248-249. https://doi.org/10.1109/ICKII.2018.8569126 